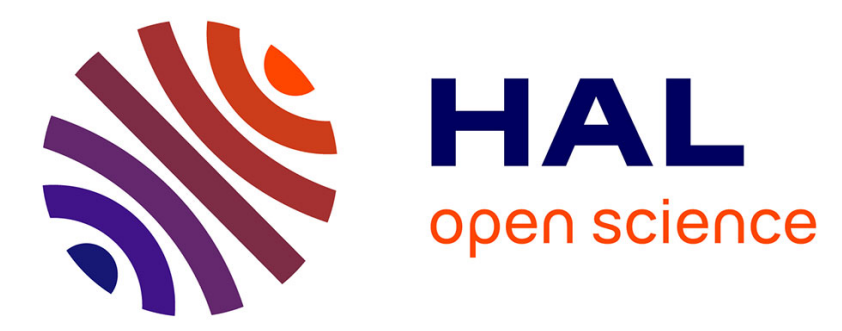

\title{
A Continuum Model for Deformable, Second Gradient Porous Media Partially Saturated with Compressible Fluids
}

\author{
Angela Madeo, Francesco Dell'Isola, Félix Darve
}

\section{- To cite this version:}

Angela Madeo, Francesco Dell'Isola, Félix Darve. A Continuum Model for Deformable, Second Gradient Porous Media Partially Saturated with Compressible Fluids. Journal of the Mechanics and Physics of Solids, 2013, 61 (11), pp.2196-2211. hal-00843626

\section{HAL Id: hal-00843626 \\ https://hal.science/hal-00843626}

Submitted on 12 Jul 2013

HAL is a multi-disciplinary open access archive for the deposit and dissemination of scientific research documents, whether they are published or not. The documents may come from teaching and research institutions in France or abroad, or from public or private research centers.
L'archive ouverte pluridisciplinaire HAL, est destinée au dépôt et à la diffusion de documents scientifiques de niveau recherche, publiés ou non, émanant des établissements d'enseignement et de recherche français ou étrangers, des laboratoires publics ou privés. 


\title{
A Continuum Model for Deformable, Second Gradient Porous Media Partially Saturated with Compressible Fluids
}

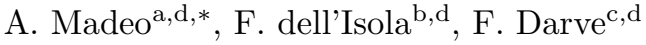 \\ ${ }^{a}$ Université de Lyon INSA, LGCIE,20 Av. Albert Einstein, 69621 Villeurbanne CEDEX, France \\ ${ }^{b}$ Dip. di Ingegneria Strutturale e Geotecnica, Università di Roma "La Sapienza", via Eudossiana 18, 00184, Rome, Italy \\ ${ }^{c}$ Institut Polytechnique de Grenoble, UJF, CNRS, Lab 3SR, BP53, 38041 Grenoble, France \\ ${ }^{d}$ International Research Center MEMOCS, University of L'Aquila, Cisterna di Latina, Italy
}

\begin{abstract}
In this paper a general set of equations of motion and duality conditions to be imposed at macroscopic surfaces of discontinuity in partially saturated, solid-second gradient porous media are derived by means of the Least Action Principle. The need of using a second gradient (of solid displacement) theory is shown to be necessary to include in the model effects related to gradients of porosity. The proposed governing equations include, in addition to balance of linear momentum for a second gradient porous continuum and to balance of water and air chemical potentials, the equations describing the evolution of solid and fluid volume fractions as supplementary independent kinematical fields. The presented equations are general in the sense that they are all written in terms of a macroscopic potential $\Psi$ which depends on the introduced kinematical fields and on their space and time derivatives. These equations are suitable to describe the motion of a partially saturated, second gradient porous medium in the elastic and hyper-elastic regime. In the second part of the paper an additive decomposition for the potential $\Psi$ is proposed which allows for describing some particular constitutive behaviours of the considered medium. While the potential associated to the solid matrix deformation is chosen in the form proposed by Cowin and Nunziato in [15], [33] and the potentials associated to water and air compressibility are chosen to assume a simple quadratic form, the macroscopic potentials associated to capillarity phenomena between water and air have to be derived with some additional considerations. In particular, two simple examples of microscopic distributions of water and air are considered: that of spherical bubbles and that of coalesced tubes of bubbles. Both these cases are suitable to describe capillarity phenomena in porous media which are close to the saturation state. Finally, an example of a simple microscopic distribution of water and air giving rise to a macroscopic capillary potential depending on the second gradient of fluid displacement is presented, showing the need of a further generalization of the proposed theoretical framework accounting for fluid second gradient effects.
\end{abstract}

Key words: Partially saturated media, Second gradient theories, Least Action Principle, Macroscopic capillary potentials for quasi saturated media

\footnotetext{
${ }^{*}$ Corresponding author

Email addresses: angela.madeo@uniroma1.it (A. Madeo), francesco.dellisola@uniroma1.it (F. dell'Isola), felix.darve@grenoble-inp.fr (F. Darve)
} 


\section{Introduction}

It is now well recognized that the question of multiphase coupling is crucial for many application fields in geomechanics, petroleum engineering, and generally for geo-environmental problems. However, any complete mathematical model is yet available, taking into account the general interactions between a solid phase, a liquid phase and a third gaseous phase. A considerable effort has been made in this sense by e.g. [23, 24, 25] in which macroscopic balance equations for multi-phase systems are derived starting from suitable average procedures in the framework of continuum irreversible thermodynamics. In the quoted papers, the presence of contact surfaces between different phases is taken into account at the microscopic level, so that the averaged macroscopic model allows for some discontinuities and singularities of basic kinematical fields. The main difficulty arising when setting up macroscopic models for non-saturated media seems to be related to the fact that, while it is possible in a straightforward manner to introduce the volume of each phase, the surfaces of separation between phases (the interfaces) are not properly taken into account. Indeed, surface tension stresses are present in these interfaces and they can play a basic role in the macroscopic behaviour of the whole body (see e. g. $[1,24,25])$. For example, the capillary pressure can induce very strong apparent cohesion in an unsaturated soil as observed in the laboratory tests or in situ (see e.g. [26]), or in discrete element numerical simulations of unsaturated granular media (see e.g. [40, 41]). One of the main purposes of the present paper is to propose a first step for building a general macroscopic framework for three-phase, solid-second gradient media. However, we limit ourselves to a description in which, at macroscopic level, no concentration of physical quantities on surfaces, lines and points is allowed. This issue could be relevant when fluid material surfaces propagate from saturated regions into unsaturated ones (as e.g. in the case of phreatic level rise): to treat this case the results presented here must be generalized in the spirit of [23].

Also relevant in this context is the effort by [11] who uses a mechanical theory with strong discontinuities at a micro level to introduce an effective stress tensor at macro scale for partially saturated porous continua. Indeed, several authors (see e.g. [11] [22], [27], [32]) tried to rigorously generalize Terzaghi's concept of effective stress to the case of partially saturated media, but several questions still remain open in this sense. For example, as shown by [12] by means of suitable homogenisation procedures, the case of quasi-saturated media is the only case in which the Bishop formula (see [10]) for effective stress can be considered to be applicable and suitable generalizations in this sense are an open field of research.

In this paper, we only deal with balance equations involving the total stress of the mixture (see Eq. $\left.(13)_{1}\right)$ and we do not try here to introduce new arguments to enlighten the concept of effective stress for partially saturated media, referring to the quoted papers for this purpose.

We decide to follow a macroscopic variational approach in the spirit of [2] who limited themselves to the saturated case. Other examples of macroscopic variational principles for multi-phase continua can be found e.g. in $[20,18,19,39]$. The study of saturated porous media considered as macroscopic systems was originated by Biot (see e.g. $[3,4,5,6,7,8,9]$ ) who introduced the governing equations for such systems either by means of balance reasoning or presenting their variational formulation. While some models have been proposed in the past mainly based on thermodynamics arguments $([24,25,13,38])$, we derive here, for the non-saturated case, the bulk equations of motion (13) and the duality conditions (14) to be assigned on macroscopic surfaces of discontinuity by means of a Least Action Principle. These equations are general in the sense that they are written in terms of a generic potential $\Psi$ depending on the introduced basic kinematical parameters. Some particular constitutive assumptions on the potential $\Psi$ are then made in the second part of the paper which allow for the description of isotropic, partially saturated, second gradient media. In particular, we propose some particular constitutive expressions for macroscopic capillary potentials accounting for capillarity effects between water and air at the microscopic level. We do not consider here capillarity phenomena due to interactions between the solid and the aqueous or the gaseous phases. In other words, we particularize the obtained general equations to the case of quasi-saturated media, in which only the water-air capillarity plays an important role. We postpone to further studies the conception of constitutive expressions for macroscopic potentials allowing for the description of microscopic solid-water and solid-air capillarity effects which are known to be important when the gaseous phase is preponderant with respect to the aqueous one (pendular regime).

The present paper is organized as follows. First the kinematics relations and mass balance equations are 
recalled for a three-phase porous medium in a general Lagrangian formalism, including the jump equations at the interfaces. Then, by imposing that the first variation of the Action functional is vanishing, the general governing equations and associated duality conditions for unsaturated porous media are obtained. Some explicit constitutive assumptions particularize the model to make it applicable to cases of interest. From here, the body is assumed to be close to the saturation state and thus, the gas is present in the medium under the form of bubbles (spherical bubbles and tubes of coalesced bubbles), allowing to obtain two different expressions for the macroscopic capillary potential. Hence, a macroscopic solid potential for isotropic, second gradient solids with voids (of the type used by Cowin and Nunziato in [15]) accounting for the deformation of the solid phase is proposed, together with a solid and a fluid potential accounting for the compressibility of the aqueous and the gaseous phase. The solid potential proposed here also account for second gradient (of solid displacement) terms which are directly related to gradients of porosity. The general governing equations and associated duality conditions derived in this paper are then explicitly particularized to the considered constitutive choices. It is well known that second gradient theories, as well as more general theories as micropolar or micromorphic ones, may be of help when one wants to describe some specific effects of microstructure on the macroscopic mecahnical behavior of considered system (see e.g. [42, 34, 43]). These generalized macroscopic theories usually introduce characteristic lengths which are related to the underlying microstructure and which can also be found by homogenisation of systems which are heterogeneous at the microscopic scale (see e.g. [16, 17]).

Finally, in section 8, a simple illustrating example is treated which allows for the description of a particular non-homogeneous microscopic distribution of air inside the REV, namely the case of truncated cones of coalesced bubbles. This example is able to enlighten the fact that, when distributions of water and air which are heterogeneous at the microscopic level are considered, then even the general theory presented here should be enriched to account for macroscopic gradients of fluid density. This means, in other words, that a second gradient (of fluid displacement) theory should be envisaged also for the fluid constituent in order to be able to treat such examples of microscopically heterogeneous distributions of the fluid phases.

\section{Kinematics and balances of mass for quasi-saturated porous media}

\subsection{Kinematics of a partially saturated porous medium}

In this section, we establish the kinematical fields that need to be introduced to describe the deformation of a solid porous matrix, on the one hand, and the flow of two fluids (e.g. water and air) through its interconnected pores, on the other hand. To this purpose, let $B_{s}, B_{w}$ and $B_{a}$ be three open subsets of $\mathbb{R}^{3}$ (usually referred to as the Lagrangian configurations of the three constituents) and let $(0, T)$ be a time interval. It is then possible to introduce the maps

$$
\chi_{s}: B_{s} \times(0, T) \rightarrow \mathbb{R}^{3}, \quad \chi_{w}: B_{w} \times(0, T) \rightarrow \mathbb{R}^{3}, \quad \chi_{a}: B_{a} \times(0, T) \rightarrow \mathbb{R}^{3}
$$

which represent the placement of the solid constituent, of water and air respectively. Moreover, let $\phi_{w}$ : $B_{s} \times(0, T) \rightarrow B_{w}$ and $\phi_{a}: B_{s} \times(0, T) \rightarrow B_{a}$ be the maps which locate, for any instant $t \in(0, T)$, the fluid material particles $\mathbf{X}_{w}$ and $\mathbf{X}_{a}$ which are in contact with the solid material particle $\mathbf{X}_{s}$. The introduced maps are related by $\phi_{w}=\chi_{w}^{-1} \circ \chi_{s}, \phi_{a}=\chi_{a}^{-1} \circ \chi_{s}$. We also assume that $\chi_{s}, \phi_{w}$ and $\phi_{a}$ are piecewise $C^{1}$ diffeomorphisms, i.e. they are continuous in $B_{s}$, while their space gradients $\nabla \boldsymbol{\chi}_{s}, \nabla \phi_{w}$ and $\nabla \phi_{a}$ are continuous a.e. in $B_{s}$ except on a surface $S_{s}$ which is assumed to be fixed in the solid-Lagrangian configuration. This means that the fields $\nabla \chi_{s}, \nabla \phi_{w}$ and $\nabla \phi_{a}$ suffer a jump at the surface $S_{s}$. Moreover, we assume that $\chi_{s}\left(B_{s}, t\right)=\chi_{w}\left(B_{w}, t\right)=\chi_{a}\left(B_{a}, t\right)$ and we denote $B_{e}(t)$ this time-varying sub-domain of $\mathbb{R}^{3}$ usually referred to as Eulerian configuration of the partially saturated porous system. We consider the three fields $\chi_{s}, \phi_{w}$ and $\phi_{a}$ to be the three independent placement fields needed to uniquely determine the placement of a macroscopic partially saturated porous system in the 3D Euclidean space (see Fig.1). Indeed, it is worth noticing that the introduced kinematical fields are macroscopic fields which do not account for the microscopic distribution of water and air inside the REV. These microscopic informations are instead contained in the constitutive choice of the macroscopic capillary potentials as it will be shown in the following. 


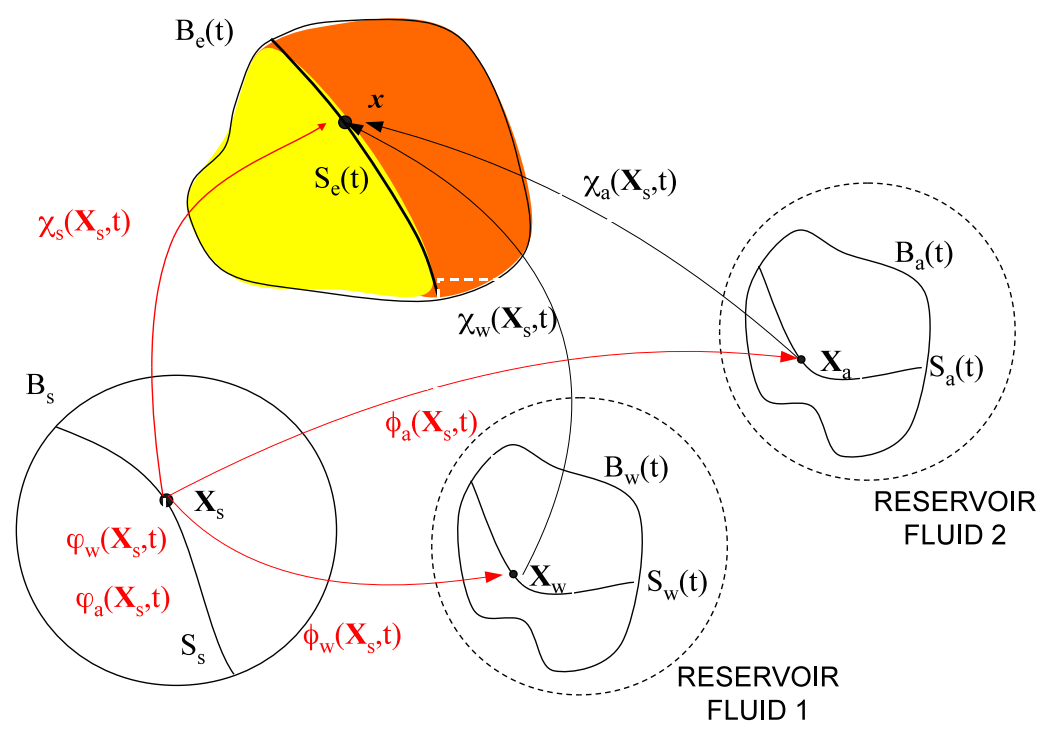

Figure 1: Kinematics of a partially saturated porous medium. The independent fields we choose to determine the motion of the considered system are the three placements $\chi_{s}, \phi_{w}$ and $\phi_{a}$ and the volume fractions of the two fluids $\varphi_{w}$ and $\varphi_{a}$.

Notation 1. Given four fields $e(\mathbf{x}, t), s\left(\mathbf{X}_{s}, t\right), w\left(\mathbf{X}_{w}, t\right)$ and $a\left(\mathbf{X}_{a}, t\right)$ defined on $B_{e}, B_{s}, B_{w}$ and $B_{a}$ respectively, we denote the corresponding transported fields as $e^{(s}:=e \circ \chi_{s}, e^{\circledR}:=e \circ \chi_{w}, e^{@}:=e \circ \chi_{a}$, $s^{\circledR}:=s \circ \boldsymbol{\phi}_{w}^{-1}, s^{\circledR}:=s \circ \boldsymbol{\phi}_{a}^{-1}, s^{\circledR}:=s \circ \boldsymbol{\chi}_{s}^{-1}, w^{\circledR}:=w \circ \boldsymbol{\chi}_{w}^{-1}, w^{\circledR}:=w \circ \boldsymbol{\phi}_{w}, w^{\circledR}:=w \circ\left(\boldsymbol{\chi}_{w}^{-1} \circ \boldsymbol{\chi}_{a}\right)$, $a^{\circledR}:=a \circ \chi_{a}^{-1}, a^{\circledR}:=a \circ \phi_{a}$ and $a^{\circledR}:=a \circ\left(\chi_{a}^{-1} \circ \chi_{w}\right)$.

In the following we set

$$
\mathbf{F}_{s}:=\nabla \chi_{s}, \quad \mathbf{G}_{w}:=\nabla \phi_{w}, \quad \mathbf{G}_{a}:=\nabla \phi_{a}, \quad J_{s}:=\operatorname{det} \mathbf{F}_{s}, \quad I_{w}:=\operatorname{det} \mathbf{G}_{w}, \quad I_{a}:=\operatorname{det} \mathbf{G}_{a}
$$

To complete the kinematical description of the considered partially saturated medium, we also introduce, on the solid-Lagrangian configuration $B_{s}$, two additional kinematical parameters accounting for the time evolution of the volume fractions of the two fluids (see Fig.1)

$$
\varphi_{a}: B_{s} \times(0, T) \rightarrow \mathbb{R}, \quad \varphi_{w}: B_{s} \times(0, T) \rightarrow \mathbb{R}
$$

The solid volume fraction $\varphi_{s}$ can be obtained from the volume fractions of the two fluids simply considering that $\varphi_{a}+\varphi_{w}+\varphi_{s}=J_{s}$. The Eulerian volume fractions $n_{w}, n_{a}$ and $n_{s}$ (defined on $B_{e}(t)$ ) can be deduced from the Lagrangian ones by means of the simple formulas $n_{w}=\left(\varphi_{w} J_{s}^{-1}\right) \Subset, n_{a}=\left(\varphi_{a} J_{s}^{-1}\right)^{\oplus}$ and $n_{s}=\left(\varphi_{s} J_{s}^{-1}\right){ }^{\circledR}$, so that it is easy to check that $n_{w}+n_{a}+n_{s}=1$.

The fact of considering the volume fractions of water and air as independent kinematical parameters allows for an accurate description of the evolution of porosity and of the degree of saturation inside the considered porous medium. Indeed, what is currently done in continuum poromechanics (see e.g. [13], [14], [20], [39]) is to assume that only the solid and fluid placements are independent kinematical fields, while porosity is usually indirectly calculated by means of so-called "saturation hypotesis" according to which porosity is obtained as the ratio between the "apparent" density of the fluid (which is indeed one of the unknowns of classical poromechanical models) and the "true" or "intrinsic" density (which is usually arbitrarily assigned and is not an unknown field of the problem). If this hypothesis is sensible in the case of saturated media, it constitutes a too strict constraint for partially saturated ones. Adding the water and 
air volume fractions as additional kinematical parameters allows for obtaining two supplementary evolution equations for $\varphi_{w}$ and $\varphi_{a}$ which are suitable to determine the fluid and gaseous volume fractions during the motion of the system. Since the classical equations giving the evolutions of water and air apparent densities are also available, the evolution of the true densities of each constituent can be indirectly obtained as the the ratio of apparent density and of volume fraction of considered constituent. To the authors' knowledge, models accounting for the different constituents volume fractions as independent kinematical parameters are available for solids with voids (see e.g. [15]), but not for porous solids totally or partially saturated with fluids.

We suppose that the introduced maps $\chi_{s}, \phi_{w}$ and $\phi_{a}$ are Piecewise $C^{1}$ diffeomorphisms, i.e. they are continuous everywhere in $B_{s}$, while their space gradients $\nabla \chi_{s}, \nabla \phi_{w}$ and $\nabla \phi_{a}$ are continuous and differentiable almost everywhere in $B_{s}$ except on a surface of discontinuity $S_{s}$. In other words, we can say that the gradients of the three considered fields suffer a jump on the surface $S_{s}$. With these regularity assumptions it is possible to prove (see [20] for extended calculations) that the following identities hold in the bulk

$$
\operatorname{div}\left(J_{s} \mathbf{F}_{s}^{-T}\right)=0, \quad \operatorname{div}\left(I_{w} \mathbf{G}_{w}^{-T}\right)=0, \quad \operatorname{div}\left(I_{a} \mathbf{G}_{a}^{-T}\right)=0,
$$

together with their surface counterpart on the surface $S_{s}$

$$
\left[\left|J_{s} \mathbf{F}_{s}^{-T} \cdot \mathbf{N}_{s}\right|\right]=0, \quad\left[\left|I_{w} \mathbf{G}_{w}^{-T} \cdot \mathbf{N}_{s}\right|\right]=0, \quad\left[\left|I_{a} \mathbf{G}_{a}^{-T} \cdot \mathbf{N}_{s}\right|\right]=0
$$

where $\mathbf{N}_{s}$ is the unit normal vector to the surface $S_{s}$ and where we denote, for any tensor field $a$ defined on the surface $S_{s}$, by $[|a|]$ the jump of the field $a$ defined as $[|a|]=a^{+}-a^{-}$.

We also remark that, using the chain rule, the following kinematical identities hold true (see also [20] for detailed calculations)

$$
\begin{gathered}
\mathbf{v}_{w}^{\Im}=\mathbf{v}_{s}-\mathbf{F}_{s} \cdot \mathbf{G}_{w}^{-1} \cdot \dot{\boldsymbol{\phi}}_{w}, \quad \mathbf{v}_{a}^{\Im}=\mathbf{v}_{s}-\mathbf{F}_{s} \cdot \mathbf{G}_{a}^{-1} \cdot \dot{\boldsymbol{\phi}}_{a} . \\
\frac{\partial}{\partial t} \mathbf{v}_{w}^{\Im}=\gamma_{w}^{\Im}+\nabla \mathbf{v}_{w}^{\Im} \cdot \mathbf{G}_{w}^{-1} \cdot \dot{\boldsymbol{\phi}}_{w}, \quad \frac{\partial}{\partial t} \mathbf{v}_{a}^{\Im}=\gamma_{a}^{\circledR}+\nabla \mathbf{v}_{a}^{(} \cdot \mathbf{G}_{a}^{-1} \cdot \dot{\boldsymbol{\phi}}_{a},
\end{gathered}
$$

with $\gamma_{w}:=\partial \mathbf{v}_{w} / \partial t, \gamma_{a}:=\partial \mathbf{v}_{a} / \partial t$ and where from now on a superposed dot indicates partial differentiation with respect to time. Finally, owing to the regularity of the considered fields, it can also be proven that (see [20] for details)

$$
\frac{\partial}{\partial t}\left(\mathbf{F}_{s} \cdot \mathbf{G}_{i}^{-1}\right)=\nabla\left(\mathbf{F}_{s} \cdot \mathbf{G}_{i}^{-1}\right) \cdot \mathbf{G}_{i}^{-1} \cdot \dot{\boldsymbol{\phi}}_{i}+\nabla \mathbf{v}_{i}^{\circledR} \cdot \mathbf{G}_{i}^{-1}, \quad i=w, a
$$

\subsection{Balances of mass}

Let $\eta_{s}, \eta_{w}$ and $\eta_{a}$ be the constant Lagrangian densities defined on $B_{s}, B_{w}$ and $B_{a}$ respectively and let $m_{w}:=I_{w} \eta_{w}$ and $m_{a}:=I_{a} \eta_{a}$ be the solid-Lagrangian apparent densities of the two fluids. The balances of mass of the three constituents (solid, water and air) in the solid-Lagrangian reference frame respectively read (see [20])

$$
\frac{\partial \eta_{s}}{\partial t}=0, \quad \frac{\partial m_{w}}{\partial t}+\operatorname{div}\left(\mathbf{D}_{w}\right)=0, \quad \frac{\partial m_{a}}{\partial t}+\operatorname{div}\left(\mathbf{D}_{a}\right)=0
$$

where we set $\mathbf{D}_{w}:=m_{w} \mathbf{F}_{s}^{-1} \cdot\left(\mathbf{v}_{w}^{\Im}-\mathbf{v}_{s}\right)$ and $\mathbf{D}_{a}:=m_{a} \mathbf{F}_{s}^{-1} \cdot\left(\mathbf{v}_{a}^{\Im}-\mathbf{v}_{s}\right)$. It is worth noticing that assuming (with no loss of generality) that the solid Lagrangian apparent density of the solid $\eta_{s}$ is constant implies that the solid balance of mass (first of Eqs. (6)) is automatically satisfied. As for the fluid balances of mass at the solid Lagrangian discontinuity surface $S_{s}$ they read

$$
\left[\left|\mathbf{D}_{w} \cdot \mathbf{N}_{s}\right|\right]=0, \quad\left[\left|\mathbf{D}_{a} \cdot \mathbf{N}_{s}\right|\right]=0
$$




\section{Action functional for a class of partially saturated porous media and its first variation}

The Lagrangian function $\mathcal{L}$ of the considered system is written, as usual, as the difference between the kinetic energy density $\Lambda$ and the strain energy density $\Psi$. In formulas

$$
\mathcal{L}=\Lambda-\Psi
$$

The expression which we consider in this paper for the kinetic energy density of the system is given by

$$
\Lambda=\frac{1}{2} m_{a}\left(\mathbf{v}_{a}^{\circledR}\right)^{2}+\frac{1}{2} m_{w}\left(\mathbf{v}_{w}^{\circledR}\right)^{2}+\frac{1}{2} \eta_{s} \mathbf{v}_{s}^{2}+\frac{1}{2} c_{w} \dot{\varphi}_{w}^{2}+\frac{1}{2} c_{a} \dot{\varphi}_{a}^{2}+c \dot{\varphi}_{w} \dot{\varphi}_{a},
$$

where the coefficients $c_{w}, c_{a}$ and $c$ have not been introduced before and are assumed to be constant in the remainder of this paper. While the first three terms appearing in the considered expression for kinetic energy are related to macroscopic inertia effects, the last three terms may be related to the microstructure of considered system (see e.g. [2]).

As for the expression of the strain energy density, we assume that it depends on the introduced kinematical fields as follows

$$
\Psi=\Psi\left(\varepsilon, \nabla \varepsilon, m_{w}, m_{a}, \varphi_{w}, \varphi_{a}, \nabla \varphi_{w}, \nabla \varphi_{a}\right),
$$

where $\varepsilon$ is the standard Green-Lagrange deformation tensor defined as

$$
\varepsilon=\frac{\mathbf{F}_{s}^{T} \cdot \mathbf{F}_{s}-\mathbf{I}}{2}
$$

We are considering an expression for the volume strain energy density $\Psi$ of the considered medium which accounts for the dependence on the second gradient of solid displacement $\nabla \varepsilon$ and on gradient of the two fluids volume fractions. As it will be better pointed out in the remainder of this paper, the fact of considering a dependence of $\Psi$ on the second gradient of solid displacement is mandatory when simultaneously considering a dependence on the gradients of water and fluid volume fractions (or indirectly on the gradient of solid volume fraction). This fact does not seem to be sufficiently clarified in the literature, for example in [15], even if a dependence on the gradient of solid volume fraction is considered, the consequent necessary dependence on the second gradient of displacement is not underlined. This has not consequences for the case considered there, since it is restricted to the linear elastic case in which the second gradient effects related to the gradient of solid volume fraction are negligible.

The action functional which we introduce to describe the motion of considered system is then naturally introduced as

$$
\mathcal{A}=\int_{\mathbb{B}_{s}} \mathcal{L} d \mathbb{B}_{s}=\int_{\mathbb{B}_{s}}(\Lambda-\Psi) d \mathbb{B}_{s},
$$

where $\mathbb{B}_{s}:=B_{s} \times[0, T]$. The variation of the action functional can be computed as

$$
\delta \mathcal{A}=\int_{\mathbb{B}_{s}} \delta \mathcal{L} d \mathbb{B}_{s}=\int_{\mathbb{B}_{s}}(\delta \Lambda-\delta \Psi) d \mathbb{B}_{s}
$$

This choice of the action functional leads to models which generalize those presented by Coussy in [14] since we are considering that the introduced functional depends on two additional kinematical parameters (and on their space and time derivatives) representing the water and air volume fractions. Moreover, the results presented here are more general because they account for the dependence of the strain energy $\Psi$ on the second gradient of solid displacement. However, as it will be seen in the following section 8, it is easy to conceive physical situations in which even the complicated theoretical framework we present here is too restrictive. 
Starting from Eq. (11) with expressions (8) and (9) for the kinetic and potential energy respectively, performing a suitable number of integration by parts, using some kinematical identities together with the balances of masses, it can be shown that (see Appendix A for detailed calculations)

$$
\begin{aligned}
& \int_{\mathbb{B}_{s}} \delta \mathcal{A}=\int_{\mathbb{B}_{s}}\left[\operatorname{div}\left[\mathbf{F}_{s} \cdot\left(\frac{\partial \Psi}{\partial \varepsilon}-\operatorname{div}\left(\frac{\partial \Psi}{\partial \nabla \varepsilon}\right)\right)\right]-\left(\eta_{s} \gamma_{s}+m_{w} \gamma_{w}^{(}+m_{a} \gamma_{a}^{(}\right)\right] \cdot \delta \boldsymbol{\chi}_{s} \\
& +\int_{\mathbb{B}_{s}} m_{w} \mathbf{G}_{w}^{-T} \cdot\left[\nabla\left(\frac{\partial \Psi}{\partial m_{w}}\right)+\gamma_{w}^{\Im} \cdot \mathbf{F}_{s}\right] \cdot \delta \boldsymbol{\phi}_{w}+\int_{\mathbb{B}_{s}} m_{a} \mathbf{G}_{a}^{-T} \cdot\left[\nabla\left(\frac{\partial \Psi}{\partial m_{a}}\right)+\gamma_{a}^{\Im} \cdot \mathbf{F}_{s}\right] \cdot \delta \boldsymbol{\phi}_{a} \\
& -\int_{\mathbb{B}_{s}}\left[\frac{\partial \Psi}{\partial \varphi_{w}}-\operatorname{div}\left(\frac{\partial \Psi}{\partial \nabla \varphi_{w}}\right)+c_{w} \ddot{\varphi}_{w}+c \ddot{\varphi}_{a}\right] \delta \varphi_{w}-\int_{\mathbb{B}_{s}}\left[\frac{\partial \Psi}{\partial \varphi_{a}}-\operatorname{div}\left(\frac{\partial \Psi}{\partial \nabla \varphi_{a}}\right)+c_{a} \ddot{\varphi}_{a}+c \ddot{\varphi}_{w}\right] \delta \varphi_{a} \\
& -\int_{\mathbb{S}_{s}}\left[\left|\left\{\left(\mathbf{F}_{s} \cdot\left(\frac{\partial \Psi}{\partial \varepsilon}-\operatorname{div}\left(\frac{\partial \Psi}{\partial \nabla \varepsilon}\right)\right)-\mathbf{v}_{w}^{\circledR} \otimes \mathbf{D}_{w}-\mathbf{v}_{a}^{\circledR} \otimes \mathbf{D}_{a}\right) \cdot \mathbf{N}_{s}-\operatorname{div}^{S}\left(\mathbf{F}_{s} \cdot \frac{\partial \Psi}{\partial \nabla \varepsilon} \cdot \mathbf{N}_{s}\right)\right\} \cdot \delta \boldsymbol{\chi}_{s}\right|\right] \\
& -\int_{\mathbb{S}_{s}}\left[\left|\left\{\left(\mathbf{F}_{s} \cdot \frac{\partial \Psi}{\partial \nabla \boldsymbol{\varepsilon}} \cdot \mathbf{N}_{s}\right) \cdot \mathbf{N}_{s}\right\} \cdot\left(\delta \boldsymbol{\chi}_{s}\right)_{n}\right|\right] \\
& +\int_{\mathbb{S}_{s}}\left[\left|m_{w} \mathbf{G}_{w}^{-T} \cdot\left(-\frac{\partial \Psi}{\partial m_{w}} \mathbf{N}_{s}+\frac{1}{2}\left(\mathbf{v}_{w}^{(s}\right)^{2} \mathbf{N}_{s}-\frac{1}{m_{w}} \mathbf{F}_{s}^{T} \cdot \mathbf{v}_{w}^{\Im} \otimes \mathbf{D}_{w} \cdot \mathbf{N}_{s}\right) \cdot \delta \boldsymbol{\phi}_{w}\right|\right] \\
& +\int_{\mathbb{S}_{s}}\left[\left|m_{a} \mathbf{G}_{a}^{-T} \cdot\left(-\frac{\partial \Psi}{\partial m_{a}} \mathbf{N}_{s}+\frac{1}{2}\left(\mathbf{v}_{a}^{\Im}\right)^{2} \mathbf{N}_{s}-\frac{1}{m_{a}} \mathbf{F}_{s}^{T} \cdot \mathbf{v}_{a}^{\circledR} \otimes \mathbf{D}_{a} \cdot \mathbf{N}_{s}\right) \cdot \delta \boldsymbol{\phi}_{a}\right|\right] \\
& -\int_{\mathbb{S}_{s}}\left[\left|\frac{\partial \Psi}{\partial \nabla \varphi_{w}} \cdot \mathbf{N}_{s} \delta \varphi_{w}\right|\right]-\int_{\mathbb{S}_{s}}\left[\left|\frac{\partial \Psi}{\partial \nabla \varphi_{a}} \cdot \mathbf{N}_{s} \delta \varphi_{a}\right|\right]-\sum_{i=1}^{N} \int_{\mathcal{E}_{i}}\left[\left|\left\{\left(\mathbf{F}_{s} \cdot \frac{\partial \Psi}{\partial \nabla \boldsymbol{\varepsilon}} \cdot \mathbf{N}_{s}\right) \cdot \boldsymbol{\nu}_{i}\right\} \cdot \delta \boldsymbol{\chi}_{s}\right|\right],
\end{aligned}
$$

where we recall that $d i v^{S}$ stands for the divergence operator on the surface $S_{s},\left(\delta \chi_{s}\right)_{n}$ stands for the normal derivative of the field $\delta \chi_{s}, \mathcal{E}_{i}, \quad i=1,2, \ldots N$ are the edges of the surface $S_{s}$ (if any) and, if the edge $\mathcal{E}_{i}$ is regarded as the border of a surface, then $\boldsymbol{\nu}_{i}$ is the normal vector to the considered edge which is tangent to the surface itself. Moreover, we also recall that, with a slight abuse of notations, we denoted with the same symbol the jump across a surface and the jump across an edge.

\section{Macroscopic equations of motion and associated jump conditions}

\subsection{General framework}

Starting from expression (12) for the variation of the action functional, imposing $\delta \mathcal{A}$ to be vanishing and considering arbitrary (in the bulk) test functions $\delta \chi_{s}, \delta \phi_{w}, \delta \phi_{a}, \delta \varphi_{w}$ and $\delta \varphi_{a}$, we get the following strong 
form of the equations of motion for a partially saturated porous medium

$$
\begin{gathered}
\operatorname{div}\left[\mathbf{F}_{s} \cdot\left(\frac{\partial \Psi}{\partial \varepsilon}-\operatorname{div}\left(\frac{\partial \Psi}{\partial \nabla \varepsilon}\right)\right)\right]=\eta_{s} \gamma_{s}+m_{w} \gamma_{w}^{\Im}+m_{a} \gamma_{a}^{\Im} \\
\nabla\left(\frac{\partial \Psi}{\partial m_{w}}\right)+\mathbf{F}_{s}^{T} \cdot \gamma_{w}^{\Im}=0, \\
\frac{\partial \Psi}{\partial \varphi_{w}}-\operatorname{div}\left(\frac{\partial \Psi}{\partial \nabla m_{a}}\right)+\mathbf{F}_{s}^{T} \cdot \gamma_{a}^{\Im}=0, \\
=-c_{w} \ddot{\varphi}_{w}-c \ddot{\varphi}_{a}, \quad \frac{\partial \Psi}{\partial \varphi_{a}}-\operatorname{div}\left(\frac{\partial \Psi}{\partial \nabla \varphi_{a}}\right)=-c_{a} \ddot{\varphi}_{a}-c \ddot{\varphi}_{w} .
\end{gathered}
$$

We explicitly remark that we obtain the same number of equations as the number of independent kinematical fields we have introduced at the beginning of the paper. This means that each of these equations represent the balance of the internal actions expending power on the virtual variation of each independent kinematical field. In particular, the first equation is the balance of internal forces (or of linear momentum) expending power on the virtual solid displacement. The second and third equations represent the balance of water and air chemical potential respectively, expending power on the relative displacement of each of the two fluids with respect to the solid phase. The last two equations represent the balance of those internal actions expending power on the water and air volume fractions virtual variations respectively. These last equations are what Cowin and Nunziato ([15], [33]) call the balance of equilibrated forces. We can additionally say that these equations may be interpreted as the balance of those internal actions which act on the change of water and air volume inside the pores, i.e. indirectly on the change of porosity.

Recalling the continuity of the quantities $m_{w} \mathbf{G}_{w}^{-T}$ and $m_{a} \mathbf{G}_{a}^{-T}$ through the discontinuity surface (Eqs. (2)), we also get the following duality conditions valid on $S_{s}$

$$
\begin{aligned}
& {\left[\mid\left\{\left(\mathbf{F}_{s} \cdot\left(\frac{\partial \Psi}{\partial \varepsilon}-\operatorname{div}\left(\frac{\partial \Psi}{\partial \nabla \varepsilon}\right)\right)-\mathbf{v}_{w}^{\circledR} \otimes \mathbf{D}_{w}-\mathbf{v}_{a}^{\subseteq} \otimes \mathbf{D}_{a}\right) \cdot \mathbf{N}_{s}-\operatorname{div} S\left(\mathbf{F}_{s} \cdot \frac{\partial \Psi}{\partial \nabla \boldsymbol{\varepsilon}} \cdot \mathbf{N}_{s}\right)\right\} \cdot \delta \boldsymbol{\chi}_{s} \|\right]=0} \\
& {\left[\left|\left\{\left(\mathbf{F}_{s} \cdot \frac{\partial \Psi}{\partial \nabla \boldsymbol{\varepsilon}} \cdot \mathbf{N}_{s}\right) \cdot \mathbf{N}_{s}\right\} \cdot\left(\delta \boldsymbol{\chi}_{s}\right)_{n}\right|\right]=0} \\
& \left.\left[\mid\left(-\frac{\partial \Psi}{\partial m_{w}} \mathbf{N}_{s}+\frac{1}{2}\left(\mathbf{v}_{w}^{(}\right)\right)^{2} \mathbf{N}_{s}-\mathbf{F}_{s}^{T} \cdot \mathbf{v}_{w}^{\Im} \otimes \frac{\mathbf{D}_{w}}{m_{w}} \cdot \mathbf{N}_{s}\right) \cdot \delta \boldsymbol{\phi}_{w} \mid\right]=0 \\
& {\left[\left|\left(-\frac{\partial \Psi}{\partial m_{a}} \mathbf{N}_{s}+\frac{1}{2}\left(\mathbf{v}_{a}^{\Im}\right)^{2} \mathbf{N}_{s}-\mathbf{F}_{s}^{T} \cdot \mathbf{v}_{a}^{\circledR} \otimes \frac{\mathbf{D}_{a}}{m_{a}} \cdot \mathbf{N}_{s}\right) \cdot \delta \boldsymbol{\phi}_{a}\right|\right]=0} \\
& {\left[\left|\frac{\partial \Psi}{\partial \nabla \varphi_{w}} \cdot \mathbf{N}_{s} \delta \varphi_{w}\right|\right]=0, \quad\left[\left|\frac{\partial \Psi}{\partial \nabla \varphi_{a}} \cdot \mathbf{N}_{s} \delta \varphi_{a}\right|\right]=0}
\end{aligned}
$$

and the following duality conditions valid on the edges (if any) of considered surface:

$$
\left[\left|\left\{\left(\mathbf{F}_{s} \cdot \frac{\partial \Psi}{\partial \nabla \boldsymbol{\varepsilon}} \cdot \mathbf{N}_{s}\right) \cdot \boldsymbol{\nu}_{i}\right\} \cdot \delta \boldsymbol{\chi}_{s}\right|\right]=0 .
$$

Imposing that the first variation of the action functional (power of internal forces) is vanishing, we are implicitly assuming (via the principle of virtual powers) that the power of external forces is vanishing as well. In other words, we are considering that no external (body or surface forces) act on the considered system, but the generalization of the obtained equations including such external actions is straightforward and can be found e.g. in [20]. 


\section{Constitutive assumptions for microscopically homogeneous, partially saturated porous me- dia}

In this section we introduce some constitutive assumptions for the solid and fluid macroscopic energy potentials. We assume that the global strain energy potential can be decomposed as

$$
\begin{aligned}
\Psi\left(\varepsilon, \nabla \varepsilon, m_{w}, m_{a}, \varphi_{w}, \varphi_{a}, \nabla \varphi_{w}, \nabla \varphi_{a}\right)= & \Psi_{s}\left(\varepsilon, \varphi_{s}, \nabla \varphi_{s}\right)+\Psi_{w}\left(m_{w}, \varphi_{w}\right)+\Psi_{a}\left(m_{a}, \varphi_{a}\right) \\
& +\Psi_{\text {capill }}\left(m_{w}, \varphi_{w}, m_{a}, \varphi_{a}\right)
\end{aligned}
$$

where $\varphi_{s}:=J_{s}-\varphi_{w}-\varphi_{a}$. It could seem that, in the considered constitutive expression, $\Psi$ does not explicitly depend on the gradient of deformation $\nabla \varepsilon$. Indeed, the dependence of $\Psi$ on the gradient of deformation is introduced by means of the dependence of $\Psi_{s}$ on $\nabla \varphi_{s}$. In fact, since $\varphi_{s}=J_{s}-\varphi_{w}-\varphi_{a}$, then $\nabla \varphi_{s}=\nabla J_{s}-\nabla \varphi_{w}-\nabla \varphi_{a}$ (remark that $\nabla J_{s}$ is related to $\nabla \varepsilon$ ). It seems then unavoidable that if one considers constitutive laws for the strain potential depending on $\nabla \varphi_{s}$ then a second gradient (for the solid displacement) theory is needed in order to have a coherent set of governing equations. Constitutive assumptions for the solid potential $\Psi_{s}$ analogous to those presented here are made in [15], [33], where the authors derive the governing equations of an elastic solid with voids on the basis of thermodynamics arguments. Nevertheless, even if the results we obtain are in agreement with theirs for what concerns first gradient terms, the authors do not underline the need of adding a second gradient stress tensor to generalize the Piola-Kirchhoff stress tensor appearing in the balance of linear momentum. If this is sensible in the case of small deformations (since, as it will be shown in Eqs. (25), the additional second gradient terms appearing in the balance of linear momentum are all quadratic in the gradient of displacement), this is not the case when considering finite strains.

Using the constitutive assumption (15), we now calculate all the partial derivatives appearing in equations (13) and (14), in order to get a simplified form for them. In order to calculate these partial derivatives, it has to be taken in mind that the independent variables of the considered problem have been chosen to be $m_{w}, m_{a}, \varphi_{w}, \varphi_{a}, \varepsilon, \nabla \varepsilon$, while an additional dependent variable, $\varphi_{s}$ (and its gradient) appears in the constitutive assumption (15). This additional variable and its space gradient are respectively related to the basic variables by the relationships (see also Appendix B)

$$
\varphi_{s}=\sqrt{\operatorname{det}(\mathbf{C})}-\varphi_{w}-\varphi_{a}, \quad\left(\varphi_{s}\right)_{, k}=\frac{1}{2} C_{i j}^{-T} C_{i j, k},
$$

were we set $\mathbf{C}:=\mathbf{F}_{s}^{T} \cdot \mathbf{F}_{s}$ and where we denoted by $C_{i j}$ its components.

Once the set of independent and dependent variables has been made clear and the constitutive choice (15) has been adopted, we can compute all the partial derivatives appearing in equations (13) and (14) as follows ${ }^{1}$

$$
\begin{array}{cc}
\frac{\partial \Psi}{\partial \varepsilon}=\frac{\partial \Psi_{s}}{\partial \varepsilon}+\frac{\partial \Psi_{s}}{\partial \varphi_{s}} \frac{\partial \varphi_{s}}{\partial \varepsilon}, & \frac{\partial \Psi}{\partial \nabla \varepsilon}=\frac{\partial \Psi_{s}}{\partial \nabla \varphi_{s}} \cdot \frac{\partial \nabla \varphi_{s}}{\partial \nabla \varepsilon}, \\
\frac{\partial \Psi}{\partial m_{w}}=\frac{\partial \Psi_{w}}{\partial m_{w}}+\frac{\partial \Psi_{\text {capill }}}{\partial m_{w}}, & \frac{\partial \Psi}{\partial m_{a}}=\frac{\partial \Psi_{a}}{\partial m_{a}}+\frac{\partial \Psi_{\text {capill }}}{\partial m_{a}} \\
\frac{\partial \Psi}{\partial \varphi_{w}}=\frac{\partial \Psi_{w}}{\partial \varphi_{w}}+\frac{\partial \Psi_{\text {capill }}}{\partial \varphi_{w}}+\frac{\partial \Psi_{s}}{\partial \varphi_{s}} \frac{\partial \varphi_{s}}{\partial \varphi_{w}}, & \frac{\partial \Psi}{\partial \varphi_{a}}=\frac{\partial \Psi_{a}}{\partial \varphi_{a}}+\frac{\partial \Psi_{\text {capill }}}{\partial \varphi_{a}}+\frac{\partial \Psi_{s}}{\partial \varphi_{s}} \frac{\partial \varphi_{s}}{\partial \varphi_{a}} \\
\frac{\partial \Psi}{\partial \nabla \varphi_{w}}=\frac{\partial \Psi_{s}}{\partial \nabla \varphi_{s}} \cdot \frac{\partial \nabla \varphi_{s}}{\partial \nabla \varphi_{w}}, & \frac{\partial \Psi}{\partial \nabla \varphi_{a}}=\frac{\partial \Psi_{s}}{\partial \nabla \varphi_{s}} \cdot \frac{\partial \nabla \varphi_{s}}{\partial \nabla \varphi_{a}} .
\end{array}
$$

\footnotetext{
${ }^{1}$ Here and in the sequel, given any $n$-th order and $k$-th order tensors $\mathbf{A}$ and $\mathbf{B}$ of components $a_{i_{1}, \ldots, i_{n}}$ and $b_{j_{1}, \ldots, j_{k}}$ respectively, we denote by $\partial \mathbf{A} / \partial \mathbf{B}$ the $n+k$-th order tensor the component of which are given by $(\partial \mathbf{A} / \partial \mathbf{B})_{i_{1}, \ldots, i_{n}, j_{1}, \ldots j_{k}}:=$ $\left(\partial a_{i_{1}, \ldots, i_{n}}\right) /\left(\partial b_{i_{1}, \ldots, i_{k}}\right)$.
} 
We then introduce more particular expressions for the single potentials $\Psi_{w}, \Psi_{a}$ and $\Psi_{\text {capill }}$ which can be suitable to model physical situations in which water and air are homogeneously distributed inside the REV (i.e. at the microscopic level). To do so, we consider the following particular forms for the macroscopic energy densities associated to the two fluids and to capillarity effects

$$
\begin{gathered}
\Psi_{w}\left(m_{w}, \varphi_{w}\right)=e_{w}\left(\frac{m_{w}}{\varphi_{w}}\right) \varphi_{w}, \quad \Psi_{a}\left(m_{a}, \varphi_{a}\right)=e_{a}\left(\frac{m_{a}}{\varphi_{a}}\right) \varphi_{a} \\
\Psi_{\text {capill }}\left(m_{w}, m_{a}, \varphi_{w}, \varphi_{a}\right)=e_{\text {capill }}\left(\frac{m_{w}}{\varphi_{w}}, \frac{m_{a}}{\varphi_{a}}\right) \varphi_{a} .
\end{gathered}
$$

The functions $e_{w}, e_{a}$ and $e_{\text {capill }}$ can be interpreted as the microscopic energies of water and air and as the microscopic capillary energy respectively when considering a homogeneous distribution of water and air inside the REV. More precisely, if the microscopic densities of water and air $\rho_{w}^{\text {micro }}$ and $\rho_{a}^{\text {micro }}$ are both constant inside each REV, this means that, denoting by $\langle\cdot\rangle$ the average of a microscopic quantity in a REV, we can write $\rho_{w}^{\text {micro }}=\left\langle\rho_{w}^{\text {micro }}\right\rangle=\rho_{w} / n_{w}=m_{w} / \varphi_{w}$ and $\rho_{a}^{\text {micro }}=\left\langle\rho_{a}^{\text {micro }}\right\rangle=\rho_{a} / n_{a}=m_{a} / \varphi_{a}$ (where $\rho_{w}:=m_{w} J_{s}^{-1}$ and $\rho_{a}:=m_{a} J_{s}^{-1}$ denote the Eulerian apparent densities of water and air respectively). It is then clear that, with these assumption of homogeneity inside the REV, the microscopic quantities $\rho_{w}^{\text {micro }}$ and $\rho_{a}^{\text {micro }}$ can be confused with the macroscopic ones $m_{w} / \varphi_{w}$ and $m_{a} / \varphi_{a}$. It is in this sense that, in the considered case of homogeneous distribution of water and air inside each REV, the potentials $e_{w}, e_{a}$ and $e_{\text {capill }}$ appearing in Eqs. (17) and (18) can be interpreted as microscopic potentials.

With the additional constitutive assumptions (17) and (18), the partial derivatives (16) read

$$
\begin{gathered}
\frac{\partial \Psi}{\partial \varepsilon}=\frac{\partial \Psi_{s}}{\partial \varepsilon}+\frac{\partial \Psi_{s}}{\partial \varphi_{s}} \frac{\partial \varphi_{s}}{\partial \varepsilon}, \quad \frac{\partial \Psi}{\partial \nabla \varepsilon}=\frac{\partial \Psi_{s}}{\partial \nabla \varphi_{s}} \cdot \frac{\partial \nabla \varphi_{s}}{\partial \nabla \varepsilon} \\
\frac{\partial \Psi}{\partial m_{w}}=\frac{\partial e_{w}}{\partial\left(m_{w} / \varphi_{w}\right)}+\frac{\varphi_{a}}{\varphi_{w}} \frac{\partial e_{\text {capill }}}{\partial\left(m_{w} / \varphi_{w}\right)}, \quad \frac{\partial \Psi}{\partial m_{a}}=\frac{\partial e_{a}}{\partial\left(m_{a} / \varphi_{a}\right)}+\frac{\partial e_{\text {capill }}}{\partial\left(m_{a} / \varphi_{a}\right)} \\
\frac{\partial \Psi}{\partial \varphi_{w}}=e_{w}-\frac{m_{w}}{\varphi_{w}} \frac{\partial e_{w}}{\partial\left(m_{w} / \varphi_{w}\right)}-\frac{\varphi_{a}}{\varphi_{w}} \frac{m_{w}}{\varphi_{w}} \frac{\partial e_{\text {capill }}}{\partial\left(m_{w} / \varphi_{w}\right)}+\frac{\partial \Psi_{s}}{\partial \varphi_{s}} \frac{\partial \varphi_{s}}{\partial \varphi_{w}} \\
\frac{\partial \Psi}{\partial \varphi_{a}}=e_{a}-\frac{m_{a}}{\varphi_{a}} \frac{\partial e_{a}}{\partial\left(m_{a} / \varphi_{a}\right)}+e_{\text {capill }}-\frac{m_{a}}{\varphi_{a}} \frac{\partial e_{\text {capill }}}{\partial\left(m_{a} / \varphi_{a}\right)}+\frac{\partial \Psi_{s}}{\partial \varphi_{s}} \frac{\partial \varphi_{s}}{\partial \varphi_{a}} \\
\frac{\partial \Psi}{\partial \nabla \varphi_{w}}=\frac{\partial \Psi_{s}}{\partial \nabla \varphi_{s}} \cdot \frac{\partial \nabla \varphi_{s}}{\partial \nabla \varphi_{w}}, \quad \frac{\partial \Psi}{\partial \nabla \varphi_{a}}=\frac{\partial \Psi_{s}}{\partial \nabla \varphi_{s}} \cdot \frac{\partial \nabla \varphi_{s}}{\partial \nabla \varphi_{a}} .
\end{gathered}
$$

\section{Up-scaling for macroscopic capillary potentials}

In this subsection we focus on finding some specific macroscopic potentials related to capillarity effects and which take the general form given in Eq. (18). To do so, we show an up-scaling procedure which allows us to present two examples of macroscopic capillary energy potentials of the type (18) corresponding to two different microscopic distributions of the air inside the REVs.

\subsection{The case of spherical bubbles}

We consider here the case of spherical air bubbles dispersed inside the interstitial water. We assume that the air mass is distributed among equal bubbles, having same mass and radius. We suppose in this case 
that the microscopic densities of water and air $\rho_{w}^{\text {micro }}$ and $\rho_{a}^{\text {micro }}$ are both constant inside each REV. This means that $\rho_{w}^{\text {micro }}=\left\langle\rho_{w}^{\text {micro }}\right\rangle=\rho_{w} / n_{w}=m_{w} / \varphi_{w}$ and $\rho_{a}^{\text {micro }}=\left\langle\rho_{a}^{\text {micro }}\right\rangle=\rho_{a} / n_{a}=m_{a} / \varphi_{a}$. With these assumptions and denoting by $u_{w}\left(\rho_{w}^{\text {micro }}\right)$ and $u_{a}\left(\rho_{a}^{\text {micro }}\right)$ the microscopic pressures of water and air inside the REV, the Laplace law can be written as

$$
\frac{2 \gamma}{R_{a}}=u_{a}\left(\frac{\rho_{a}}{n_{a}}\right)-u_{w}\left(\frac{\rho_{w}}{n_{w}}\right) \Longrightarrow R_{a}\left(\rho_{a}, n_{a}, \rho_{w}, n_{w}\right)=\frac{2 \gamma}{u_{a}-u_{w}}
$$

where $\gamma$ is the surface tension of the water-air interface, $R_{a}$ is the radius of air bubbles. It is known that in the case of spherical bubbles $u_{a}>u_{w}$ so that $u_{a}-u_{w}>0$.

Let $N_{a}$ be the number of air bubbles per unit of Eulerian volume $d B_{e}$, then the volume of air per unit of Eulerian volume $d B_{e}$ (air volume fraction $n_{a}$ ) is

$$
n_{a}=N_{a} \frac{4}{3} \pi R_{a}^{3} \Longrightarrow N_{a}=\frac{3 n_{a}}{4 \pi R_{a}^{3}}
$$

The surface of air per unit of Eulerian volume is $4 \pi R_{a}^{2} N_{a}$ so that the macroscopic Eulerian energy potential can be written as $W_{\text {capill }}=\gamma 4 \pi R_{a}^{2} N_{a}$ which, replacing the obtained expressions for $N_{a}$ and $R_{a}$, simplifies into

$$
W_{\text {capill }}=\frac{3}{2} n_{a}\left(u_{a}\left(\frac{m_{a}}{\varphi_{a}}\right)-u_{w}\left(\frac{m_{w}}{\varphi_{w}}\right)\right) .
$$

The corresponding solid-Lagrangian potential $\Psi_{\text {capill }}$ is easily computed as

$$
\Psi_{\text {capill }}\left(m_{w}, m_{a}, \varphi_{w}, \varphi_{a}\right)=J_{s} W_{\text {capill }}=\frac{3}{2}\left(u_{a}\left(\frac{m_{a}}{\varphi_{a}}\right)-u_{w}\left(\frac{m_{w}}{\varphi_{w}}\right)\right) \varphi_{a}
$$

which is in the form (18) when setting $e_{\text {capill }}=3 / 2\left(u_{a}-u_{w}\right)$.

\subsection{The case of tubes of coalesced bubbles}

Also in this case we suppose that water and air are homogeneously distributed inside each REV, we can write $\rho_{w}^{\text {micro }}=\left\langle\rho_{w}^{\text {micro }}\right\rangle=\rho_{w} / n_{w}$ and $\rho_{a}^{\text {micro }}=\left\langle\rho_{a}^{\text {micro }}\right\rangle=\rho_{a} / n_{a}$. With these assumptions, denoting by $u_{w}\left(\rho_{w}^{\text {micro }}\right)$ and $u_{a}\left(\rho_{a}^{\text {micro }}\right)$ the microscopic pressures of water and air inside the REV, the Laplace law can be written as

$$
\frac{\gamma}{R_{a}}=u_{a}\left(\frac{\rho_{a}}{n_{a}}\right)-u_{w}\left(\frac{\rho_{w}}{n_{w}}\right) \Longrightarrow R_{a}\left(\rho_{a}, n_{a}, \rho_{w}, n_{w}\right)=\frac{\gamma}{u_{a}-u_{w}}
$$

$R_{a}$ is the radius of the cylinder of coalesced bubbles in a REV. Let $N_{a}$ be the number of air cylinders per unit of Eulerian volume $d B_{e}$, then the volume of air per unit of Eulerian volume (Eulerian air volume fraction $\left.n_{a}\right)$ is

$$
n_{a}=N_{a} \pi R_{a}^{2} L \Longrightarrow N_{a}=\frac{n_{a}}{\pi R_{a}^{2} L}
$$

where $L$ is the length of the cylinder. The surface of air per unit of Eulerian volume is $2 \pi R_{a} L N_{a}$ and the Eulerian macroscopic capillary energy is then $W_{\text {capill }}=\gamma 2 \pi R_{a} L N_{a}$, which replacing the calculated values of $N_{a}$ and $R_{a}$ gives

$$
W_{\text {capill }}=2 n_{a}\left(u_{a}\left(\frac{m_{a}}{\varphi_{a}}\right)-u_{w}\left(\frac{m_{w}}{\varphi_{w}}\right)\right) .
$$

The corresponding solid Lagrangian capillary potential is easily evaluated as

$$
\Psi_{\text {capill }}\left(m_{w}, m_{a}, \varphi_{w}, \varphi_{a}\right)=J_{s} W_{\text {capill }}=2\left(u_{a}\left(\frac{m_{a}}{\varphi_{a}}\right)-u_{w}\left(\frac{m_{w}}{\varphi_{w}}\right)\right) \varphi_{a},
$$

which is in the form (18) when setting $e_{\text {capill }}=2\left(u_{a}-u_{w}\right)$. 


\section{Governing equations for isotropic, microscopically homogeneous, partially saturated porous media}

In this section we further particularize the obtained set of equations of motion and naturally associated boundary conditions to treat the simplified case of elastic and isotropic solid deformation and almost incompressible fluids. These simplified equations are a suitable basis to study e.g. wave propagation in partially saturated media. To do so, following Cowin and Nunziato [15], we introduce a particular expression for the potential $\Psi_{s}$ of the considered porous matrix which is valid in the linear-isotropic, elastic case:

$$
\Psi_{s}\left(\varepsilon, \varphi_{s}, \nabla \varphi_{s}\right)=\mu \varepsilon\left|\varepsilon+\frac{\lambda}{2}(\operatorname{tr} \varepsilon)^{2}+\beta \varphi_{s} t r \varepsilon+\frac{1}{2} \zeta \varphi_{s}^{2}+\frac{1}{2} \alpha \nabla \varphi_{s}\right| \nabla \varphi_{s}
$$

where the elasticity parameters $\lambda, \mu, \alpha, \beta$ and $\zeta$ are all assumed to be constant. Moreover, we choose the following quadratic expressions for the microscopic energies of water and air respectively

$$
\begin{array}{r}
e_{w}\left(\frac{m_{w}}{\varphi_{w}}\right)=\frac{1}{2} K_{w}\left(\frac{m_{w}}{\varphi_{w}}-\frac{m_{w}^{0}}{\varphi_{w}^{0}}\right)^{2}+C_{w}\left(\frac{m_{w}}{\varphi_{w}}-\frac{m_{w}^{0}}{\varphi_{w}^{0}}\right), \\
e_{a}\left(\frac{m_{a}}{\varphi_{a}}\right)=\frac{1}{2} K_{a}\left(\frac{m_{a}}{\varphi_{a}}-\frac{m_{a}^{0}}{\varphi_{a}^{0}}\right)^{2}+C_{a}\left(\frac{m_{a}}{\varphi_{a}}-\frac{m_{a}^{0}}{\varphi_{a}^{0}}\right) .
\end{array}
$$

With this choice of the water and air potentials, we are assuming that the water and air densities can only have small variations during the deformation of the porous system.

As for the capillary potential, we have

$$
e_{\text {capill }}\left(\frac{m_{w}}{\varphi_{w}}, \frac{m_{a}}{\varphi_{a}}\right)=K_{\text {capill }}\left(u_{a}\left(\frac{m_{a}}{\varphi_{a}}\right)-u_{w}\left(\frac{m_{w}}{\varphi_{w}}\right)\right),
$$

where $K_{\text {capill }}=3 / 2$ for the case of spherical bubbles or $K_{\text {capill }}=2$ for the case of tubes of coalesced bubbles (see previous section). Moreover, the pressures $u_{w}$ and $u_{a}$ of water and air, can be evaluated from the microscopic potentials according the common thermodynamics relations between pressure and free energy:

$$
u_{w}=-e_{w}+\frac{m_{w}}{\varphi_{w}} \frac{\partial e_{w}}{\partial\left(m_{w} / \varphi_{w}\right)}, \quad u_{a}=-e_{a}+\frac{m_{a}}{\varphi_{a}} \frac{\partial e_{a}}{\partial\left(m_{a} / \varphi_{a}\right)} .
$$

Using the particular expressions (20), (21) and (22) for the introduced energy potentials and recalling 
equations (23), it can be checked that ${ }^{2}$

$$
\begin{gathered}
\frac{\partial \Psi_{s}}{\partial \varepsilon}=2 \mu \varepsilon+\left(\lambda \operatorname{tr} \varepsilon+\beta \varphi_{s}\right) \mathbf{I}, \quad \frac{\partial \Psi_{s}}{\partial \varphi_{s}} \frac{\partial \varphi_{s}}{\partial \varepsilon}=\left(\beta \operatorname{tr} \varepsilon+\zeta \varphi_{s}\right) J_{s} \mathbf{C}^{-T} \\
\frac{\partial \Psi}{\partial \varphi_{s}} \frac{\partial \varphi_{s}}{\partial \varphi_{w}}=\frac{\partial \Psi_{s}}{\partial \varphi_{s}} \frac{\partial \varphi_{s}}{\partial \varphi_{a}}=-\left(\beta \operatorname{tr} \varepsilon+\zeta \varphi_{s}\right) \\
\frac{\partial \Psi_{s}}{\partial \nabla \varphi_{s}} \cdot \frac{\partial \nabla \varphi_{s}}{\partial \nabla \varphi_{w}}=\frac{\partial \Psi_{s}}{\partial \nabla \varphi_{s}} \cdot \frac{\partial \nabla \varphi_{s}}{\partial \nabla \varphi_{a}}=-\alpha \nabla \varphi_{s}, \quad \frac{\partial \Psi_{s}}{\partial \nabla \varphi_{s}} \cdot \frac{\partial \nabla \varphi_{s}}{\partial \nabla \varepsilon}=\alpha J_{s} \mathbf{C}^{-T} \otimes \nabla \varphi_{s} \\
\frac{\partial e_{w}}{\partial\left(m_{w} / \varphi_{w}\right)}=K_{w}\left(\frac{m_{w}}{\varphi_{w}}-\frac{m_{w}^{0}}{\varphi_{w}^{0}}\right)+C_{w}, \quad \frac{\partial e_{a}}{\partial\left(m_{a} / \varphi_{a}\right)}=K_{a}\left(\frac{m_{a}}{\varphi_{a}}-\frac{m_{a}^{0}}{\varphi_{a}^{0}}\right)+C_{a}, \\
\frac{\partial e_{\text {capill }}}{\partial\left(m_{w} / \varphi_{w}\right)}=-K_{\text {capill }} K_{w} \frac{m_{w}}{\varphi_{w}}, \quad \frac{\partial e_{\text {capill }}}{\partial\left(m_{a} / \varphi_{a}\right)}=K_{\text {capill }} K_{a} \frac{m_{a}}{\varphi_{a}} .
\end{gathered}
$$

\footnotetext{
${ }^{2}$ To get some of these equalities, we used the fact that $\partial \varphi_{s} / \partial \varphi_{a}=\partial \varphi_{s} / \partial \varphi_{w}=-1, \partial \nabla \varphi_{s} / \partial \nabla \varphi_{a}=\partial \nabla \varphi_{s} / \partial \nabla \varphi_{w}=-\mathbf{I}$, $\partial \varphi_{s} / \partial \varepsilon=\partial J_{s} / \partial \varepsilon, \partial\left(\nabla \varphi_{s}\right) /(\partial \nabla \varepsilon)=\partial\left(\nabla J_{s}\right) /(\partial \nabla \varepsilon)$, so that it can be checked that (see Appendix B for calculations)

$$
J_{s}=\sqrt{\operatorname{det}(2 \varepsilon+\mathbf{I})} \Longrightarrow \frac{\partial J_{s}}{\partial \varepsilon}=J_{s} \mathbf{C}^{-T} \text { and } \frac{\partial\left(J_{s}\right)_{, k}}{\partial \varepsilon_{l m, n}}=J_{s} C_{l m}^{-1} \delta_{k n} .
$$
}


Using equations (19) and (24) and simplifying ${ }^{3}$, the equations of motion (13) can be finally rewritten as

$$
\begin{aligned}
& \operatorname{div}\left\{\mathbf{F}_{s} \cdot\left[2 \mu \varepsilon+\left(\lambda \operatorname{tr} \varepsilon+\beta \varphi_{s}\right) \mathbf{I}\right]+\left[\beta \operatorname{tr} \varepsilon+\zeta \varphi_{s}\right] J_{s} \mathbf{F}_{s}^{-T}\right\} \\
& -\operatorname{div}\left\{\left[\operatorname{div}\left(\alpha \nabla \varphi_{s}\right) \mathbf{I}+\left(\frac{\alpha}{2} \nabla \varphi_{s} \cdot\left(\mathbf{C}^{-1}: \nabla \mathbf{C}\right)\right) \mathbf{I}-J_{s} \alpha\left(\mathbf{F}_{s}^{-T} \cdot \nabla \mathbf{C} \cdot \nabla \varphi_{s}\right) \cdot \mathbf{F}^{-1}\right] \cdot J_{s} \mathbf{F}_{s}^{-T}\right\} \\
& =\eta_{s} \gamma_{s}+m_{w} \gamma_{w}^{\circledR}+m_{a} \gamma_{a}^{\circledR}, \\
& \nabla\left[K_{w}\left(\frac{m_{w}}{\varphi_{w}}-\frac{m_{w}^{0}}{\varphi_{w}^{0}}\right)-\frac{\varphi_{a}}{\varphi_{w}} K_{\text {capill }} K_{w} \frac{m_{w}}{\varphi_{w}}\right]+\mathbf{F}_{s}^{T} \cdot \gamma_{w}^{\circledR}=0, \\
& \nabla\left[K_{a}\left(\frac{m_{a}}{\varphi_{a}}-\frac{m_{a}^{0}}{\varphi_{a}^{0}}\right)+K_{\text {capill }} K_{a} \frac{m_{a}}{\varphi_{a}}\right]+\mathbf{F}_{s}^{T} \cdot \gamma_{a}^{\circledR}=0, \\
& -\left(\beta \operatorname{tr} \varepsilon+\zeta \varphi_{s}\right)+\operatorname{div}\left(\alpha \nabla \varphi_{s}\right)+e_{w}-\frac{m_{w}}{\varphi_{w}}\left(K_{w}\left(\frac{m_{w}}{\varphi_{w}}-\frac{m_{w}^{0}}{\varphi_{w}^{0}}\right)+C_{w}\right) \\
& +\frac{\varphi_{a}}{\varphi_{w}}\left(\frac{m_{w}}{\varphi_{w}}\right)^{2} K_{\text {capill }} K_{w}=-c_{w} \ddot{\varphi}_{w}-c \ddot{\varphi}_{a}, \\
& -\left(\frac{m_{a}}{\varphi_{a}}\right)^{2} K_{\text {capill }} K_{a}=-c_{a} \ddot{\varphi}_{a}-c \ddot{\varphi}_{w}, \\
& -\left(\beta \operatorname{tr} \varepsilon+\zeta \varphi_{s}\right)+\operatorname{div}\left(\alpha \nabla \varphi_{s}\right)+e_{a}+e_{\text {capill }}-\frac{m_{a}}{\varphi_{a}}\left(K_{a}\left(\frac{m_{a}}{\varphi_{a}}-\frac{m_{a}^{0}}{\varphi_{a}^{0}}\right)+C_{a}\right)
\end{aligned}
$$

where we recall that the constitutive expressions for $e_{w}, e_{a}$ and $e_{\text {capill }}$ are given by the constitutive equations (22) and (21). We remark that, even if the expression obtained for the first of equations (25) might seem complicated, this form of the balance equations is suitable to be easily transported on the Eulerian configuration of the system thanks to the Piola transformation $J \mathbf{F}^{-T}$ inside the solid-Lagrangian divergence operator (we refer to [20] for the definition of the operations needed to transport a balance law from one configuration to the other).

Analogously, it can be checked that, always using equations (19) and (24), the boundary conditions (14) are rewritten as

\footnotetext{
${ }^{3}$ To get the first of equations (25) we also used the fact that $C_{j k, p}^{-1}=-C_{j i}^{-1} C_{i h, p} C_{h k}^{-1}$
} 


$$
\begin{aligned}
& {\left[\left|\left\{\left[\mathbf{F}_{s} \cdot\left(2 \mu \varepsilon+\left(\lambda t r \varepsilon+\beta \varphi_{s}\right) \mathbf{I}\right)+\left(\beta \operatorname{tr} \varepsilon+\zeta \varphi_{s}\right) J_{s} \mathbf{F}_{s}^{-T}\right] \cdot \mathbf{N}_{s}\right\} \cdot \delta \boldsymbol{\chi}_{s}\right|\right]} \\
& -\left[\left|\left\{\left[\operatorname{div}\left(\alpha \nabla \varphi_{s}\right) \mathbf{I}+\left(\frac{\alpha}{2} \nabla \varphi_{s} \cdot\left(\mathbf{C}^{-1}: \nabla \mathbf{C}\right)\right) \mathbf{I}-J_{s} \alpha\left(\mathbf{F}_{s}^{-T} \cdot \nabla \mathbf{C} \cdot \nabla \varphi_{s}\right) \cdot \mathbf{F}^{-1}\right] \cdot J_{s} \mathbf{F}_{s}^{-T} \cdot \mathbf{N}_{s}\right\} \cdot \delta \boldsymbol{\chi}_{s}\right|\right] \\
& -\left[\left|\left\{\left(\mathbf{v}_{w}^{\Im} \otimes \mathbf{D}_{w}+\mathbf{v}_{a}^{\Im} \otimes \mathbf{D}_{a}\right) \cdot \mathbf{N}_{s}+d i v^{S}\left(\alpha J_{s} \mathbf{F}_{s}^{-T} \otimes \nabla \varphi_{s} \cdot \mathbf{N}_{s}\right)\right\} \cdot \delta \boldsymbol{\chi}_{s}\right|\right]=0 \\
& {\left[\left|\left(\alpha J_{s} \mathbf{F}_{s}^{-T} \cdot \mathbf{N}_{s} \otimes \nabla \varphi_{s} \cdot \mathbf{N}_{s}\right) \cdot\left(\delta \chi_{s}\right)_{n}\right|\right]=0} \\
& -\left[\mid\left(\left[K_{w}\left(\frac{m_{w}}{\partial \varphi_{w}}-\frac{m_{w}^{0}}{\partial \varphi_{w}^{0}}\right)+C_{w}-\frac{\varphi_{a}}{\varphi_{w}} K_{\text {capill }} K_{w} \frac{m_{w}}{\partial \varphi_{w}}\right] \mathbf{N}_{s}\right) \cdot \delta \boldsymbol{\phi}_{w} \|\right] \\
& \left.\left.+\left[\mid\left(\frac{1}{2}\left(\mathbf{v}_{w}^{(}\right)\right)^{2} \mathbf{N}_{s}-\mathbf{F}_{s}^{T} \cdot \mathbf{v}_{w}^{(}\right) \otimes \frac{\mathbf{D}_{w}}{m_{w}} \cdot \mathbf{N}_{s}\right) \cdot \delta \boldsymbol{\phi}_{w} \mid\right]=0 \\
& -\left[\left|\left(\left[K_{a}\left(\frac{m_{a}}{\partial \varphi_{a}}-\frac{m_{a}^{0}}{\partial \varphi_{a}^{0}}\right)+C_{a}+K_{\text {capill }} K_{a} \frac{m_{a}}{\partial \varphi_{a}}\right] \mathbf{N}_{s}\right) \cdot \delta \boldsymbol{\phi}_{a}\right|\right] \\
& +\left[\left|\left(\frac{1}{2}\left(\mathbf{v}_{a}^{\Im}\right)^{2} \mathbf{N}_{s}-\mathbf{F}_{s}^{T} \cdot \mathbf{v}_{a}^{\Im} \otimes \frac{\mathbf{D}_{a}}{m_{a}} \cdot \mathbf{N}_{s}\right) \cdot \delta \boldsymbol{\phi}_{a}\right|\right]=0 \\
& {\left[\left|-\left(\alpha \nabla \varphi_{s} \cdot \mathbf{N}_{s}\right) \delta \varphi_{w}\right|\right]=0, \quad\left[\left|-\left(\alpha \nabla \varphi_{s} \cdot \mathbf{N}_{s}\right) \delta \varphi_{a}\right|\right]=0} \\
& {\left[\left|\left\{\left(\alpha J_{s} \mathbf{F}_{s}^{-T} \otimes \nabla \varphi_{s} \cdot \mathbf{N}_{s}\right) \cdot \boldsymbol{\nu}_{i}\right\} \cdot \delta \boldsymbol{\chi}_{s}\right|\right]=0 .}
\end{aligned}
$$

These duality conditions can be used to impose suitable kinematical or natural boundary conditions at macroscopic surfaces of discontinuity of the material properties of the considered medium. More particularly, depending on the physics of the problem in study, one can think to impose forces or displacement, double forces or normal derivative of displacement, chemical potentials or relative fluid-solid displacement, partial pore pressures or fluid volume fractions. Moreover, since we are dealing with a solid-second gradient theory also forces per unit line or displacements can be assigned on the edges (if any) of considered surface (last of conditions (26)). It can be remarked that the coupled system of PDEs which we obtain in this paper has a very involved structure. As already remarked, a first study should involve the characterization of the properties of waves propagating in our complex continuum: this is feasible when considering the results presented in $[21,37,36,29,30,35]$

\section{An example of fluid capillarity leading to fluid second gradient macroscopic interactions}

In this section we show a simple example of microscopic water and air distribution inside the pores which leads to a macroscopic capillary potential which is more general than the ones considered up to now. More particularly, we will show that, as far as the microscopic density distribution of one of the fluids is not homogeneous inside the REV, then a second gradient theory must be considered also for the fluid constituent. This means that some situations may exist for which the solid-second gradient macroscopic theory presented in this paper is not sufficient and should be generalized by considering a strain potential depending on the second gradient of fluid placement. We consider here a simple case of microscopic heterogeneous distribution 
of air inside the considered porous medium. In particular, we assume that the air inside each REV consists of a truncated cone of coalesced bubbles of length comparable with the size of the REV. Since we are considering a truncated cone, the section of the tube varies inside the REV, so that the microscopic pressure of air cannot be supposed to be constant in the REV itself. In particular, we assume that the microscopic density of air $\rho_{a}^{\text {micro }}$ can linearly vary inside each truncated cone as

$$
\rho_{a}^{\text {micro }}(\mathbf{y})=\frac{\rho_{a}}{n_{a}}+\nabla\left(\frac{\rho_{a}}{n_{a}}\right) \cdot\left(\mathbf{y}-\mathbf{y}_{0}\right),
$$

where $\nabla\left(\rho_{a} / n_{a}\right)$ is the space gradient of the macroscopic true density, $\mathbf{y}_{0}$ is the centroid of the REV (which also coincides with the center of the axis of the truncated cone) and $\mathbf{y}$ is a variable spanning on the axis of the truncated cone. This ansatz concerning the structure of the microscopic air density field inside any REV is the simplest possible which can be considered among the density fields such that i) they are spatially non-constant inside the REV, ii) $\left\langle\rho_{a}^{\text {micro }}\right\rangle=\rho_{a} / n_{a}$ and iii $)\left\langle\nabla \rho_{a}^{\text {micro }}\right\rangle=\nabla\left(\rho_{a} / n_{a}\right)$. On the other hand, we consider that outside the truncated cone of bubbles the microscopic density of water $\rho_{w}^{\text {micro }}$ is constant in space and equal to $\rho_{w} / n_{w}$. This implies that that the microscopic pressure $u_{w}\left(\rho_{w} / n_{w}\right)$ is also constant inside the REV. We remark that expression (27) for the microscopic density of air can be pulled back on the Lagrangian configuration recalling that $\left(\rho_{a} / n_{a}\right)^{\circledR}=m_{a} / \varphi_{a}$, that $\left(\nabla\left(\rho_{a} / n_{a}\right)\right)^{\circledR}=\nabla\left(m_{a} / \varphi_{a}\right) \cdot \mathbf{F}_{s}^{-1}$ and that $\left(\mathbf{y}-\mathbf{y}_{0}\right)=\mathbf{F}_{s} \cdot\left(\mathbf{Y}-\mathbf{Y}_{0}\right)$, where $\mathbf{Y}_{0}$ and $\mathbf{Y}$ are the centroid of the REV and a coordinate spanning on the axis of the tube in Lagrangian coordinates respectively. Indeed, it is then easy to check that equation (27) implies

$$
\rho_{a}^{\text {micro }}(\mathbf{Y})=\frac{m_{a}}{\varphi_{a}}+\nabla\left(\frac{m_{a}}{\varphi_{a}}\right) \cdot\left(\mathbf{Y}-\mathbf{Y}_{0}\right)=\frac{m_{a}}{\varphi_{a}}+\nabla\left(\frac{m_{a}}{\varphi_{a}}\right) \cdot s \mathbf{E},
$$

where $s$ is a curvilinear abscissa which spans on the axis of the truncated cone in the Lagrangian configuration. In other words, if $\mathbf{E}$ is a unit vector along the axis of the tube and if $L$ is the size of the Lagrangian REV, then $s \mathbf{E}=\mathbf{Y}-\mathbf{Y}_{0}$ for $s \in[-L / 2, L / 2]$ and for $\mathbf{Y}$ spanning on the axis of the tube.

The Laplace law for the considered case reads

$$
\frac{\gamma}{R(\mathbf{y})}=u_{a}\left(\rho_{a}^{\text {micro }}(\mathbf{y})\right)-u_{w}\left(\frac{\rho_{w}}{n_{w}}\right) \Longrightarrow R(\mathbf{y})=\frac{\gamma}{u_{a}(\mathbf{y})-u_{w}}
$$

where $R(\mathbf{y})$ is the radius of the circular sections of the truncated cone. This equation can be simply pulled back on the solid-Lagrangian configuration as

$$
\frac{\gamma}{R(\mathbf{Y})}=u_{a}\left(\rho_{a}^{\text {micro }}(\mathbf{Y})\right) u_{w}\left(\frac{m_{w}}{\varphi_{w}}\right) \Longrightarrow R(\mathbf{Y})=\frac{\gamma}{u_{a}(\mathbf{Y})-u_{w}}
$$

where $\rho_{a}^{\text {micro }}(\mathbf{Y})$ is given by Eq. (28).

We now assume that the microscopic air pressure takes the constitutive form

$$
u_{a}(\mathbf{Y})=u_{a}^{0}+c\left(\rho_{a}^{\text {micro }}(\mathbf{Y})-\rho_{a}^{0}\right)
$$

Considering that the radius of the cross section of the tube is linearly varying with $s$, i.e. $R(s)=$ $\left(R_{1}-R_{0}\right) s / L+\left(R_{1}+R_{0}\right) / 2$, where $R_{0}$ and $R_{1}$ are the radii of the circular sections of the cone for $s=-L / 2$ and $s=L / 2$ respectively, then the Laplace Law evaluated in $s=-L / 2$ and $s=L / 2$ respectively implies

$$
\begin{aligned}
& R_{0}=\frac{\gamma}{u_{a}^{0}-u_{w}-c \rho_{a}^{0}+c\left(m_{a} / \varphi_{a}-L / 2 \nabla\left(m_{a} / \varphi_{a}\right) \cdot \mathbf{E}\right)}, \\
& R_{1}=\frac{\gamma}{u_{a}^{0}-u_{w}-c \rho_{a}^{0}+c\left(m_{a} / \varphi_{a}+L / 2 \nabla\left(m_{a} / \varphi_{a}\right) \cdot \mathbf{E}\right)} .
\end{aligned}
$$


We explicitly remark that the two radii of the truncated cone explicitly depend on the macroscopic gradient of air density.

Let $N_{a}$ be the number of air truncated cones per unit of Eulerian volume $d B_{e}$, then the air volume fraction is

$$
n_{a}=N_{a} \frac{1}{3} \pi L\left(R_{0}^{2}+R_{1}^{2}+R_{0} R_{1}\right)
$$

which implies

$$
N_{a}=\frac{3 n_{a}}{\pi L\left(R_{0}^{2}+R_{1}^{2}+R_{0} R_{1}\right)}
$$

The surface of air per unit of Eulerian volume is given by the surface of the truncated cone

$$
N_{a} \pi\left(R_{0}+R_{1}\right) \sqrt{L^{2}+\left(R_{1}-R_{0}\right)^{2}}
$$

which multiplied by the surface tension $\gamma$ finally gives the macroscopic capillary energy potential

$$
W_{\text {capill }}=\frac{3 \gamma n_{a}\left(R_{0}+R_{1}\right)}{L\left(R_{0}^{2}+R_{1}^{2}+R_{0} R_{1}\right)} \sqrt{L^{2}+\left(R_{1}-R_{0}\right)^{2}}
$$

or equivalently

$$
\Psi_{\text {capill }}\left(m_{w}, \varphi_{w}, m_{a}, \varphi_{a}, \nabla m_{a}, \nabla \varphi_{a}\right)=J_{s} W_{\text {capill }}=\frac{3 \gamma \varphi_{a}\left(R_{0}+R_{1}\right)}{L\left(R_{0}^{2}+R_{1}^{2}+R_{0} R_{1}\right)} \sqrt{L^{2}+\left(R_{1}-R_{0}\right)^{2}},
$$

where $R_{0}$ and $R_{1}$ are given by (31).

We have shown in this section a simple example of non-homogeneous distribution of microscopic air density inside the REV which gives rise to a macroscopic capillary potential depending not only on the macroscopic density of water, but also on its macroscopic gradient. This means that, even in simple cases as the one shown in this section, a macroscopic capillary potential of the type used in this paper (see Eq. (18)) is not sufficient to take into account the effects of a heterogeneous distribution of water and air inside the REV. Indeed, in the simple case considered in this section a macroscopic capillary potential depending on the gradient of air density is obtained. This fact suggests that the theory presented in this paper and which seemed to be complicated due to the presence of a second gradient for the solid constituent is not general enough to take into account complex capillary interactions. A more general theory accounting for the presence of second gradient of the fluid placements $\phi_{w}$ and $\phi_{a}$ (i.e. of first gradient of the fluid densities) is needed to treat some kind of problems and can be obtained in the spirit of what done in [39].

\section{Conclusions}

A methodology has been proposed in this paper for building a mathematical model for solid-second gradient three-phase media. This methodology based on a Hamilton-type variational principle (Least Action Principle) is not novel (see e.g. [2, 20, 28, 31, 39]), but its application to unsaturated porous media is developed here for the first time (at least according to the authors knowledge). The macroscopic equations of motion have been established as well as the macroscopic jump conditions on the interfaces. By considering an additive decomposition of the strain energy potential, the three phase potentials and a capillary potential are exhibited. As for the solid potential we propose a second gradient generalization of the one used by Cowin and Nunziato in [15] for isotropic elastic porous solids with voids. The water and air potentials are chosen, in the last part of the paper, to be those of quasi-incompressible fluids, while some different simple cases have been considered for the choice of the capillary potential. Indeed, it is clear that the main difficulty of modelling three-phase media in a macroscopic framework is related to the construction of this last potential. We exhibit two macroscopic capillary potentials which are obtained from two distinct homogeneous distributions of air inside the pores: spherical bubbles and tubes of coalesced bubbles. Eventually, in a last part, a more 
complicated situation accounting for a microscopically heterogeneous distribution of air inside the REV has been treated: truncated cones of coalesced bubbles. It is worth noticing that this last case has led to macroscopic fluid second gradient effects, due to the microscopic interface coupling between water and air which are heterogeneously distributed inside the REV.

All the examples illustrated in this paper show the capabilities of the proposed model to describe the mechanical behaviour of three phase media, even if limited here to porous materials close to saturation conditions. Forthcoming works should consider the application of the presented theory to the case of wave propagation in partially saturated media (using the methods proposed in $[21,37,29,30,35]$ ) and the more general case of unsaturated assemblies of elastic spherical grains (following micro-mechanical arguments of the type presented in $[40,41])$.

\section{Acknowledgement}

One of the authors expresses his warm gratitude to the University of Roma La Sapienza for the support to a position as visiting professor. All the authors gratefully thank the International Research Center M\&MoCS for the material welcome. 


\section{A. Variation of the Action and Rayleigh Functionals}

We start noticing that, out of the singularity surface $S_{s}$ and considering suitably regular fields, the variations of the introduced auxiliary fields can be written in terms of the variations of the basic independent kinematical fields as

$$
\begin{aligned}
& \delta \varepsilon=\frac{1}{2} \delta\left(\mathbf{F}_{s}^{T} \cdot \mathbf{F}_{s}-\mathbf{I}\right)=\frac{1}{2}\left(\delta \mathbf{F}_{s}^{T} \cdot \mathbf{F}_{s}+\mathbf{F}_{s}^{T} \cdot \delta \mathbf{F}_{s}\right)=\left(\mathbf{F}_{s}^{T} \cdot \nabla\left(\delta \boldsymbol{\chi}_{s}\right)\right)^{s y m}, \\
& \delta \eta_{s}=0, \quad \delta \mathbf{v}_{s}=\delta \dot{\boldsymbol{\chi}}_{s} \\
& \delta I_{i}=\delta\left(\operatorname{det}\left(\nabla \boldsymbol{\phi}_{i}\right)\right)=I_{i} \mathbf{G}_{i}^{-T} \mid \nabla\left(\delta \boldsymbol{\phi}_{i}\right), \quad i=w, a ; \\
& \delta \eta_{i}^{\circledR}=\delta\left(\eta_{i} \circ \boldsymbol{\phi}_{i}\right)=\left(\nabla \eta_{i}\right)^{\circledR} \cdot \delta \boldsymbol{\phi}_{i}=\nabla \eta_{i}^{\circledR} \cdot \mathbf{G}_{i}^{-1} \cdot \delta \boldsymbol{\phi}_{i}, \quad i=w, a \\
& \delta m_{i}=\delta\left(I_{i} \eta_{i}^{\Im}\right)=\eta_{i}^{\Im} \delta I_{i}+I_{i} \delta \eta_{i}^{\Im}=\operatorname{div}\left(\eta_{i}^{\Im} I_{i} \mathbf{G}_{i}^{-1} \cdot \delta \boldsymbol{\phi}_{i}\right)=\operatorname{div}\left(m_{i} \mathbf{G}_{i}^{-1} \cdot \delta \boldsymbol{\phi}_{i}\right), \quad i=w, a .
\end{aligned}
$$

Recalling Eq. (3), the variations of the solid Lagrangian fluid velocities $\mathbf{v}_{i}^{(}, i=w, a$ are now computed $^{4}$

$$
\delta \mathbf{v}_{i}^{\circledR}=\delta \dot{\boldsymbol{\chi}}_{s}-\nabla\left(\delta \boldsymbol{\chi}_{s}\right) \cdot \mathbf{G}_{i}^{-1} \cdot \dot{\boldsymbol{\phi}}_{i}+\mathbf{F}_{s} \cdot \mathbf{G}_{i}^{-1} \cdot \nabla\left(\delta \boldsymbol{\phi}_{i}\right) \cdot \mathbf{G}_{i}^{-1} \cdot \dot{\boldsymbol{\phi}}_{i}-\mathbf{F}_{s} \cdot \mathbf{G}_{i}^{-1} \cdot \delta \dot{\boldsymbol{\phi}}_{i}, \quad i=w, a .
$$

Let us now perform the solid-Lagrangian variation $\delta \mathcal{A}$ of the action functional. To do so, we start computing the variation of the strain energy density which, recalling definition (9) for $\Psi$, using expressions (32) for the variations of the involved auxiliary functions and considering that the tensor $\partial \Psi / \partial \varepsilon$ is a second order symmetric tensor and that $\partial \Psi / \partial \nabla \varepsilon$ is a third order tensor symmetric with respect to its first two indexes, can be written as

$$
\begin{aligned}
\delta \Psi & =\frac{\partial \Psi}{\partial \varepsilon}\left|\delta \varepsilon+\frac{\partial \Psi}{\partial \nabla \varepsilon}\right| \delta \nabla \varepsilon+\frac{\partial \Psi}{\partial m_{w}} \delta m_{w}+\frac{\partial \Psi}{\partial m_{a}} \delta m_{a} \\
& +\frac{\partial \Psi}{\partial \varphi_{w}} \delta \varphi_{w}+\frac{\partial \Psi}{\partial \varphi_{a}} \delta \varphi_{a}+\frac{\partial \Psi}{\partial \nabla \varphi_{w}}\left|\delta \nabla \varphi_{w}+\frac{\partial \Psi}{\partial \nabla \varphi_{a}}\right| \delta \nabla \varphi_{a} \\
& =\frac{\partial \Psi}{\partial \varepsilon}\left|\left(\mathbf{F}_{s}^{T} \cdot \nabla\left(\delta \boldsymbol{\chi}_{s}\right)\right)+\frac{\partial \Psi}{\partial \nabla \varepsilon}\right| \nabla\left(\mathbf{F}_{s}^{T} \cdot\left(\nabla \delta \boldsymbol{\chi}_{s}\right)\right) \\
& +\frac{\partial \Psi}{\partial m_{w}} \operatorname{div}\left(m_{w} \mathbf{G}_{w}^{-1} \cdot \delta \boldsymbol{\phi}_{w}\right)+\frac{\partial \Psi}{\partial m_{a}} \operatorname{div}\left(m_{a} \mathbf{G}_{a}^{-1} \cdot \delta \boldsymbol{\phi}_{a}\right) \\
& +\frac{\partial \Psi}{\partial \varphi_{w}} \delta \varphi_{w}+\frac{\partial \Psi}{\partial \varphi_{a}} \delta \varphi_{a}+\frac{\partial \Psi}{\partial \nabla \varphi_{w}}\left|\nabla \delta \varphi_{w}+\frac{\partial \Psi}{\partial \nabla \varphi_{a}}\right| \nabla \delta \varphi_{a}
\end{aligned}
$$

Considering the integral form of expression (34), assuming that the considered test functions have compact support $K$ included in $B_{s}$ with non-vanishing intersection with the discontinuity surface $S_{s}$ and integrating

\footnotetext{
${ }^{4}$ Here, and everywhere else is not differently specified, we indicate with a superposed dot the partial time derivative of a solid-Lagrangian field.
} 
by parts in space a suitable number of times we get

$$
\begin{aligned}
\int_{\mathbb{B}_{s}} \delta \Psi & =-\int_{\mathbb{B}_{s}} \operatorname{div}\left[\mathbf{F}_{s} \cdot\left(\frac{\partial \Psi}{\partial \varepsilon}-\operatorname{div}\left(\frac{\partial \Psi}{\partial \nabla \boldsymbol{\varepsilon}}\right)\right)\right] \cdot \delta \boldsymbol{\chi}_{s}-\int_{\mathbb{B}_{s}}\left[m_{w} \mathbf{G}_{w}^{-T} \cdot \nabla\left(\frac{\partial \Psi}{\partial m_{w}}\right)\right] \cdot \delta \boldsymbol{\phi}_{w} \\
& -\int_{\mathbb{B}_{s}}\left[m_{a} \mathbf{G}_{a}^{-T} \cdot \nabla\left(\frac{\partial \Psi}{\partial m_{a}}\right)\right] \cdot \delta \boldsymbol{\phi}_{a}+\int_{\mathbb{B}_{s}}\left(\frac{\partial \Psi}{\partial \varphi_{w}}-\operatorname{div}\left(\frac{\partial \Psi}{\partial \nabla \varphi_{w}}\right)\right) \delta \varphi_{w}+\int_{\mathbb{B}_{s}}\left(\frac{\partial \Psi}{\partial \varphi_{a}}-\operatorname{div}\left(\frac{\partial \Psi}{\partial \nabla \varphi_{a}}\right)\right) \delta \varphi_{a} \\
& +\int_{\mathbb{S}_{s}}\left[\left|\left\{\mathbf{F}_{s} \cdot\left(\frac{\partial \Psi}{\partial \varepsilon}-\operatorname{div}\left(\frac{\partial \Psi}{\partial \nabla \varepsilon}\right)\right) \cdot \mathbf{N}_{s}\right\} \cdot \delta \boldsymbol{\chi}_{s}\right|\right]+\int_{\mathbb{S}_{s}}\left[\left|\left(\mathbf{F}_{s} \cdot \frac{\partial \Psi}{\partial \nabla \boldsymbol{\varepsilon}} \cdot \mathbf{N}_{s}\right)\right| \nabla\left(\delta \boldsymbol{\chi}_{s}\right) \mid\right] \\
& +\int_{\mathbb{S}_{s}}\left[\left|m_{w} \mathbf{G}_{w}^{-T} \cdot \frac{\partial \Psi}{\partial m_{w}} \mathbf{N}_{s} \cdot \delta \boldsymbol{\phi}_{w}\right|\right]+\int_{\mathbb{S}_{s}}\left[\left|m_{a} \mathbf{G}_{a}^{-T} \cdot \frac{\partial \Psi}{\partial m_{a}} \mathbf{N}_{s} \cdot \delta \boldsymbol{\phi}_{a}\right|\right]+ \\
& +\int_{\mathbb{S}_{s}}\left[\left|\frac{\partial \Psi}{\partial \nabla \varphi_{w}} \cdot \mathbf{N}_{s} \delta \varphi_{w}\right|\right]+\int_{\mathbb{S}_{s}}\left[\left|\frac{\partial \Psi}{\partial \nabla \varphi_{a}} \cdot \mathbf{N}_{s} \delta \varphi_{a}\right|\right]
\end{aligned}
$$

One of the surface terms of the previous sum, namely the one in which $\nabla\left(\delta \chi_{s}\right)$ appears can be integrated by parts once more by using the surface divergence theorem. More particularly, we recognize that the second order tensor $\nabla\left(\delta \chi_{s}\right)$ can be decomposed as

$$
\nabla\left(\delta \boldsymbol{\chi}_{s}\right)=\nabla\left(\delta \boldsymbol{\chi}_{s}\right) \cdot\left(\mathbf{N}_{s} \otimes \mathbf{N}_{s}\right)+\nabla\left(\delta \boldsymbol{\chi}_{s}\right) \cdot\left(\mathbf{I}-\mathbf{N}_{s} \otimes \mathbf{N}_{s}\right)=:\left(\delta \boldsymbol{\chi}_{s}\right)_{n} \otimes \mathbf{N}_{s}+\nabla^{S} \delta \boldsymbol{\chi}_{s},
$$

where we denoted by $\left(\delta \boldsymbol{\chi}_{s}\right)_{n}$ the normal derivative of $\delta \boldsymbol{\chi}_{s}$ and by $\nabla^{S} \delta \boldsymbol{\chi}_{s}$ its surface gradient. With these notations and applying the surface divergence theorem, the mentioned surface term appearing in the sum (35) can be rewritten as

$$
\begin{aligned}
& \int_{\mathbb{S}_{s}}\left[\left|\left(\mathbf{F}_{s} \cdot \frac{\partial \Psi}{\partial \nabla \boldsymbol{\varepsilon}} \cdot \mathbf{N}_{s}\right)\right| \nabla\left(\delta \boldsymbol{\chi}_{s}\right) \mid\right]=\int_{\mathbb{S}_{s}}\left[\left|\left\{\left(\mathbf{F}_{s} \cdot \frac{\partial \Psi}{\partial \nabla \boldsymbol{\varepsilon}} \cdot \mathbf{N}_{s}\right) \cdot \mathbf{N}_{s}\right\} \cdot\left(\delta \boldsymbol{\chi}_{s}\right)_{n}+\left(\mathbf{F}_{s} \cdot \frac{\partial \Psi}{\partial \nabla \boldsymbol{\varepsilon}} \cdot \mathbf{N}_{s}\right)\right| \nabla^{S} \delta \boldsymbol{\chi}_{s} \mid\right] \\
& =\int_{\mathbb{S}_{s}}\left[\left|\left\{\left(\mathbf{F}_{s} \cdot \frac{\partial \Psi}{\partial \nabla \boldsymbol{\varepsilon}} \cdot \mathbf{N}_{s}\right) \cdot \mathbf{N}_{s}\right\} \cdot\left(\delta \boldsymbol{\chi}_{s}\right)_{n}\right|\right] \\
& -\int_{\mathbb{S}_{s}}\left[\left|d i v^{S}\left(\mathbf{F}_{s} \cdot \frac{\partial \Psi}{\partial \nabla \boldsymbol{\varepsilon}} \cdot \mathbf{N}_{s}\right) \cdot \delta \boldsymbol{\chi}_{s}\right|\right]+\sum_{i=1}^{N} \int_{\mathcal{E}_{i}}\left[\left|\left\{\left(\mathbf{F}_{s} \cdot \frac{\partial \Psi}{\partial \nabla \boldsymbol{\varepsilon}} \cdot \mathbf{N}_{s}\right) \cdot \boldsymbol{\nu}_{i}\right\} \cdot \delta \boldsymbol{\chi}_{s}\right|\right]
\end{aligned}
$$

where $d i v^{S}$ represents the divergence operator restrained on the surface $S_{s}$. Moreover $\mathcal{E}_{i}, i=1, \ldots, N$ are the edges of the surface $S_{s}$ (if any) and, if the edge $\mathcal{E}_{i}$ is regarded as the border of a surface, then $\boldsymbol{\nu}_{i}$ is the normal vector to the considered edge which is tangent to the surface (notice that $\boldsymbol{\nu}_{i}$ necessarily suffers a jump through the edge $\mathcal{E}_{i}$ ). In this last formula, with a slight abuse of notation we indicate with the same notation the jump across a surface and the jump across a line. Using Eq. (36) in Eq. (35), we are then able to recover the variation of the potential energy which, with the sign changed, finally appears in the variation of the action functional (12).

As for the variation of the kinetic energy density, it is easy to show that, according to Eq. (8), it reads

$$
\begin{aligned}
\delta \Lambda & =\eta_{s} \mathbf{v}_{s} \cdot \delta \mathbf{v}_{s}+m_{w} \mathbf{v}_{w}^{\Im} \cdot \delta \mathbf{v}_{w}^{\Im}+m_{a} \mathbf{v}_{a}^{\Im} \cdot \delta \mathbf{v}_{a}^{\Im}+\frac{1}{2} \mathbf{v}_{s}^{2} \delta \eta_{s}+\frac{1}{2}\left(\mathbf{v}_{w}^{\Im}\right)^{2} \delta m_{w}+\frac{1}{2}\left(\mathbf{v}_{a}^{\Im}\right)^{2} \delta m_{a} \\
& +\left(c_{w} \dot{\varphi}_{w}+c \dot{\varphi}_{a}\right) \delta \dot{\varphi}_{w}+\left(c_{a} \dot{\varphi}_{a}+c \dot{\varphi}_{w}\right) \delta \dot{\varphi}_{a}
\end{aligned}
$$


which, recalling the variations computed in (32) and (33) of the involved auxiliary functions can be decomposed as $\delta \Lambda=\delta \Lambda_{s}+\delta \Lambda_{w}+\delta \Lambda_{a}+\delta \Lambda_{\varphi}$ with

$$
\begin{aligned}
\delta \Lambda_{s} & :=\left(\eta_{s} \mathbf{v}_{s}+m_{w} \mathbf{v}_{w}^{\Im}+m_{a} \mathbf{v}_{a}^{\Im}\right) \cdot \delta \dot{\chi}_{s}-m_{w} \mathbf{v}_{w}^{\Im} \cdot \nabla\left(\delta \boldsymbol{\chi}_{s}\right) \cdot \mathbf{G}_{w}^{-1} \cdot \dot{\boldsymbol{\phi}}_{w}-m_{a} \mathbf{v}_{a}^{\Im} \cdot \nabla\left(\delta \boldsymbol{\chi}_{s}\right) \cdot \mathbf{G}_{a}^{-1} \cdot \dot{\boldsymbol{\phi}}_{a}, \\
\delta \Lambda_{i} & :=\frac{1}{2}\left(\mathbf{v}_{i}^{\Im}\right)^{2} \operatorname{div}\left(m_{i} \mathbf{G}_{i}^{-1} \cdot \delta \boldsymbol{\phi}_{i}\right)+m_{i} \mathbf{v}_{i}^{\Im} \cdot \mathbf{F}_{s} \cdot \mathbf{G}_{i}^{-1} \cdot \nabla\left(\delta \boldsymbol{\phi}_{i}\right) \cdot \mathbf{G}_{i}^{-1} \cdot \dot{\boldsymbol{\phi}}_{i}-m_{i} \mathbf{v}_{i}^{\Im} \cdot \mathbf{F}_{s} \cdot \mathbf{G}_{i}^{-1} \cdot \delta \dot{\boldsymbol{\phi}}_{i}, \quad i=w, a, \\
\delta \Lambda_{\varphi} & :=\left(c_{w} \dot{\varphi}_{w}+c \dot{\varphi}_{a}\right) \delta \dot{\varphi}_{w}+\left(c_{a} \dot{\varphi}_{a}+c \dot{\varphi}_{w}\right) \delta \dot{\varphi}_{a} .
\end{aligned}
$$

We integrate separately the four parts of the variation of the introduced kinetic energy $\delta \Lambda_{s}, \delta \Lambda_{w}$ and $\delta \Lambda_{a}$ and $\delta \Lambda_{\varphi}$. Recalling that $\eta_{s}$ is assumed to be constant, integrating by parts in space and time, considering isochronous motions $\left(\delta \boldsymbol{\chi}_{s}(0)=\delta \boldsymbol{\chi}_{s}(T)\right)$ and setting $\mathbf{D}_{i}=-m_{i} \mathbf{G}_{i}^{-1} \cdot \dot{\boldsymbol{\phi}}_{i}, i=w, a$ we get

$$
\begin{aligned}
& \int_{\mathbb{B}_{s}} \delta \Lambda_{s}=-\int_{\mathbb{B}_{s}}\left[\eta_{s} \boldsymbol{\gamma}_{s}+m_{w} \dot{\mathbf{v}}_{w}^{\Im}+\dot{m}_{w} \mathbf{v}_{w}^{(}+\operatorname{div}\left(\mathbf{v}_{w}^{\Im} \otimes \mathbf{D}_{w}\right)+m_{a} \dot{\mathbf{v}}_{a}^{\circledR}+\dot{m}_{a} \mathbf{v}_{a}^{(}+\operatorname{div}\left(\mathbf{v}_{a}^{(} \otimes \mathbf{D}_{a}\right)\right] \cdot \delta \boldsymbol{\chi}_{s} \\
& +\int_{\mathbb{S}_{s}}\left[\left|\left(\left(\mathbf{v}_{w}^{\Im} \otimes \mathbf{D}_{w}+\mathbf{v}_{a}^{\circledR} \otimes \mathbf{D}_{a}\right) \cdot \mathbf{N}_{s}\right) \cdot \delta \boldsymbol{\chi}_{s}\right|\right] \\
& =-\int_{\mathbb{B}_{s}}\left(\eta_{s} \gamma_{s}+m_{w} \gamma_{w}^{\Im}+m_{a} \gamma_{a}^{\Im}\right) \cdot \delta \boldsymbol{\chi}_{s}+\int_{\mathbb{S}_{s}}\left[\left|\left(\left(\mathbf{v}_{w}^{\Im} \otimes \mathbf{D}_{w}+\mathbf{v}_{a}^{\circledR} \otimes \mathbf{D}_{a}\right) \cdot \mathbf{N}_{s}\right) \cdot \delta \boldsymbol{\chi}_{s}\right|\right],
\end{aligned}
$$

where expressions (3)-(4) for $\mathbf{v}_{w}^{\circledR}, \mathbf{v}_{a}^{\circledR}, \dot{\mathbf{v}}_{w}^{\circledR}$ and $\dot{\mathbf{v}}_{a}^{\circledR}$ and the balances of mass (6) for $\dot{m}_{w}$ and $\dot{m}_{a}$ have also been used to get the last equality.

We then integrate over $\mathbb{B}_{s}$ the terms $\delta \Lambda_{i}, i=w, a$. It can be recognized that integrating by parts in space and time, considering isochronous motions $\left(\delta \phi_{i}(0)=\delta \phi_{i}(T)\right)$, we have

$$
\begin{aligned}
\int_{\mathbb{B}_{s}} \delta \Lambda_{i} & =\int_{\mathbb{B}_{s}}\left[-\frac{1}{2} m_{i} \nabla\left(\left(\mathbf{v}_{i}^{\Im}\right)^{2}\right) \cdot \mathbf{G}_{i}^{-1}+\operatorname{div}\left(\mathbf{v}_{i}^{\circledR} \cdot \mathbf{F}_{s} \cdot \mathbf{G}_{i}^{-1} \otimes \mathbf{D}_{i}\right)+\frac{\partial}{\partial t}\left(m_{i} \mathbf{v}_{i}^{\circledR} \cdot \mathbf{F}_{s} \cdot \mathbf{G}_{i}^{-1}\right)\right] \cdot \delta \boldsymbol{\phi}_{i} \\
& +\int_{\mathbb{S}_{s}}\left[\left|\left(\frac{1}{2} m_{i}\left(\mathbf{v}_{i}^{\Im}\right)^{2} \mathbf{G}_{i}^{-T} \cdot \mathbf{N}_{s}-\mathbf{v}_{i}^{\Im} \cdot \mathbf{F}_{s} \cdot \mathbf{G}_{i}^{-1} \otimes \mathbf{D}_{i} \cdot \mathbf{N}_{s}\right) \cdot \delta \boldsymbol{\phi}_{i}\right|\right]
\end{aligned}
$$

It can be checked that, using equation (4) for the definition of $\gamma_{i}^{(}$, equation (5) for $\partial\left(\mathbf{F}_{s} \cdot \mathbf{G}_{s}^{-1}\right) / \partial t$, equations (6) for $\dot{m}_{i}$ and simplifying, we have for $i=w, a$

$$
\int_{\mathbb{B}_{s}} \delta \Lambda_{i}=\int_{\mathbb{B}_{s}}\left(m_{i} \gamma_{i}^{\Im} \cdot \mathbf{F}_{s} \cdot \mathbf{G}_{i}^{-1}\right) \cdot \delta \boldsymbol{\phi}_{i}+\int_{\mathbb{S}_{s}}\left[\left|\left(\frac{1}{2} m_{i} \mathbf{G}_{i}^{-T}\left(\mathbf{v}_{i}^{\Im}\right)^{2} \cdot \mathbf{N}_{s}-\mathbf{G}_{i}^{-T} \cdot \mathbf{F}_{s}^{T} \cdot \mathbf{v}_{i}^{\Im} \otimes \mathbf{D}_{i} \cdot \mathbf{N}_{s}\right) \cdot \delta \boldsymbol{\phi}_{i}\right|\right]
$$

As for the last term $\delta \Lambda_{\varphi}$ we integrate over $\mathbb{B}_{s}$, we integrate by parts in time considering isochronous motions $\left(\delta \varphi_{i}(0)=\delta \varphi_{i}(T) . i=w, a\right)$ and recalling that $c_{w}, c_{a}$ and $c$ are chosen to be constant so getting:

$$
\int_{\mathbb{B}_{s}} \delta \Lambda_{\varphi}=-\int_{\mathbb{B}_{s}}\left(c_{w} \ddot{\varphi}_{w}+c \ddot{\varphi}_{a}\right) \delta \varphi_{w}-\int_{\mathbb{B}_{s}}\left(c_{a} \ddot{\varphi}_{a}+c \ddot{\varphi}_{w}\right) \delta \varphi_{a}
$$

Starting from the obtained variations of the potential energy (Eq. (35)) and of the kinetic energy (Eqs. (38), (39) and (40)), expression (12) for the variation of the action functional for the considered partially saturated porous medium can be easily recognized.

\section{B. Some useful calculations}

Starting from definition (10) of $\varepsilon$ and recalling that $J_{s}=\operatorname{det} \mathbf{F}_{s}$ it is easy to recover that

$$
J_{s}=\sqrt{\operatorname{det}(2 \varepsilon+\mathbf{I})}
$$


Differentiating this equation with respect to $\varepsilon$ and using the chain rule, it is possible to check that

$$
\frac{\partial J_{s}}{\partial \varepsilon}=\sqrt{\operatorname{det}(2 \varepsilon+\mathbf{I})}(2 \varepsilon+\mathbf{I})^{-T}=J_{s} \mathbf{F}_{s}^{-1} \cdot \mathbf{F}_{s}^{-T}=J_{s} \mathbf{C}^{-T},
$$

where, to get the second equality, we used expression (10) for $\varepsilon$.

We finally start evaluating the gradient of $J_{s}$. We make this calculation by using indexes and, in order to lighten notations, we set here $J:=J_{s}$, we introduce the tensor $\mathbf{C}:=\mathbf{F}_{s}^{T} \cdot \mathbf{F}_{s}$ and we denote by $C_{i j}$ its components. With these notations and using the chain rule, the gradient of $J$ can be evaluated as

$$
J_{, k}=\frac{1}{2 \sqrt{\operatorname{det} \mathbf{C}}} \frac{\partial(\operatorname{det} \mathbf{C})}{\partial C_{i j}} C_{i j, k}=\frac{1}{2} \sqrt{\operatorname{det} \mathbf{C}} C_{i j}^{-T} C_{i j, k} .
$$

Recalling that $C_{i j}=2 \varepsilon_{i j}+\delta_{i j}$, it is easy to recognize that

$$
\frac{\partial C_{i j, k}}{\partial \varepsilon_{l m, n}}=2 \delta_{i l} \delta_{j m} \delta_{k n}
$$

Starting from Eq. (41), it is now possible to compute the derivative of $\nabla J$ with respect to $\nabla \varepsilon$ as follows

$$
\frac{\partial\left(J_{, k}\right)}{\partial\left(\varepsilon_{l m, n}\right)}=\frac{1}{2} J C_{i j}^{-T} \frac{\partial C_{i j, k}}{\partial \varepsilon_{l m, n}}
$$

which, using Eq. (42) implies

$$
\frac{\partial\left(J_{, k}\right)}{\partial\left(\varepsilon_{l m, n}\right)}=J C_{l m}^{-T} \delta_{k n}
$$

\section{References}

[1] Auffray N., dell'Isola F., Eremeyev V., Madeo A., Rosi G., 2013. Analytical continuum mechanics á la Hamilton-Piola: least action principle for second gradient continua and capillary fluids. Mathematics and Mechanics of Solids, Accepted.

[2] Berryman J. G. and Thigpen L., 1985. Nonlinear and semilinear dynamic poroelasticity with microstructure. J. Mech. Phys. Solids 33, 97-116.

[3] Biot M. A., 1973. Nonlinear and semilinear rheology of porous solids, J. Geophys. Res. 78, $4924-4937$.

[4] Biot M.A.: General theory of three-dimensional consolidation. J. Appl. Phys. 12, 155-164 (1941)

[5] Biot M.A.: General solutions of the equations of elasticity and consolidation for a porous material. J. Appl. Mech. 23, 91-96 (1956a)

[6] Biot M.A.: Theory of propagation of elastic waves in fluid-saturated porous solid. J. Acoust. Soc. Am. 28, 168-191 (1956b)

[7] Biot M.A., Willis D G.: The elastic coefficients of the theory of consolidation. J. Appl. Mech. 24 594-601 (1957)

[8] Biot M.A.: Mechanics of deformation and acoustic propagation in porous media. J. Appl. Phys. 33, 1482-1498 (1962)

[9] Biot M.A.: Variational principles for acoustic gravity waves. Phys. Fluids 6, 772-778 (1963)

[10] Bishop A.W., 1959. The principle of effective stress. Teknisk Ukeblad; 39, 859-863.

[11] Borja, R.I.:, 2006. On the mechanical energy and effective stress in saturated and unsaturated porous continua. Int. J. Solids Struct. 43, 1764-1786.

[12] Chateau X., Dormieux L., 2002. Micromechanics of saturated and unsaturated porous media. Int. J. Numer. Anal. Meth. Geomech. 26, 831-844.

[13] Coussy O., 1995. Mechanics of Porous Continua. Wiley, New York.

[14] Coussy O., 2004. Poromechanics, John Wiley and Sons Ltd, Chichester, England.

[15] Cowin S.C., Nunziato J.W., 1981. Linear elastic materials with voids. Journal of Elasticity. 13, 125-147

[16] dellisola F., Rosa L., Wo zniak C., 1997. Dynamics of solids with micro periodic non-connected fluid inclusions. Archive of Applied Mechanics, 67, 215-228.

[17] F. dellIsola, L. Rosa, and C. Wo zniak, 1998. A micro-structured continuum modelling compacting fluid-saturated grounds: The effects of pore-size scale parameter. Acta Mechanica, 127, 165-182.

[18] dellIsola F., Wo zniak C., 1997 . On phase transition layers in certain micro-damaged two-phase solids. International Journal of Fracture, 83, 175189, .

[19] dellisola F. and Wo zniak C., 1997. On continuum modelling the interphase layers in certain two-phase elastic solids. ZAMM , 77, 519-526.

[20] dell'Isola, F., Madeo, A., Seppecher, P., 2009. Boundary conditions at fluid-permeable interfaces in porous media: A variational approach. Int. J. Solids. Struct. 46 (17), 3150-3164.

[21] dell'Isola F., Madeo A., Placidi L., 2012. Plane wave propagation and normal transmission and reflection at discontinuity surfaces in second gradient 3D Continua. Zeitschrift fur Angewandte Mathematik und Mechanik (ZAMM), 92:1, 5271. 
[22] Gray, W.G., Schrefler, B.A., 2001. Thermodynamic approach to effective stress in partially saturated porous media. Eur. J. Mech. A/Solids 20, 521538.

[23] Gray W. G. and Hassanizadeh S. M., 1998. Macroscale continuum mechanics for multiphase porous-media flow including phases, interfaces, common lines, and common points. Advances in Water Resources 21, 261-281.

[24] Hassanizadeh, S.M., Gray, W.G., 1979. General Conservation Equations for Multi-phase Systems Mass, Momentum, Energy, and Entropy Equations. Advances in Water Resources 2, 191208.

[25] Hassanizadeh, S.M., Gray, W.G., 1990. Mechanics and thermodynamics of multiphase flow in porous media including interphase boundaries. Adv. Water Resour. 13, 169-186.

[26] Hicher P-Y, 1998. Experimental behaviour of granular materials. In Behavior of Granular Materials, Cambou B (ed.). Springer: New York, ; 197.

[27] Li, X.S., 2003. Effective stress in unsaturated soil: A microstructural analysis. Gotechnique 53, 273277.

[28] A. Madeo, F. dellIsola, N. Ianiro and G. Sciarra, 2008. A Variational Deduction of Second Gradient Poroelasticity II: an Application to the Consolidation Problem. J. Mech. Materials Struct. 3 (4), 607-625.

[29] Madeo, A., Gavrilyuk, S. L., Jan. 2010. Propagation of acoustic waves in porous media and their reflection and transmission at a pure-fluid/porous-medium permeable interface. Eur. J. Mech. A-Solids 29 (5), 897-910.

[30] Madeo A., Djeran-Maigre I., Rosi G., Silvani C., 2012. The Effect of Fluid Streams in Porous Media on Acoustic Compression Wave Propagation, Transmission and Reflection. Continuum Mechanics and Thermodynamics (6 March 2012), 1-24, doi:10.1007/s00161-012-0236-y, DOI: 007/s00161-012-0236-y.

[31] Madeo, A., George, D., Lekszycki, T., Rémond Y., 2012. A second gradient continuum model accounting for some effects of micro-structure on reconstructed bone remodelling. C. R. Mecanique, 340:8, 575589.

[32] Nikooee E., Habibagahi G., Hassanizadeh S.M., Ghahramani A., 2012. The Effective Stress in Unsaturated Soils: Insights from Thermodynamics. in Unsuturated Soils: Research and Applications, Part 1, 5-11.

[33] Nunziato J.W., Cowin S.C. 1979. A Nonlinear Theory of Elastic Materials with Voids. Arch. Rational Mech. Anal., 72, $175-201$.

[34] Pietraszkiewicz W., Eremeyev V.A., 2009. On natural strain measures of the non-linear micropolar continuum. International Journal of Solids and Structures, 46:3, 774-787.

[35] Placidi L., Rosi G., Giorgio I., Madeo A., 2013. Reflection and transmission of plane waves at surfaces carrying material properties and embedded in second gradient materials. Mathematics and Mechanics of Solids, DOI: $10.1177 / 1081286512474016$.

[36] Romeo F., Luongo A., 2002. Invariant representation of propagation properties for bi-coupled periodic structures. Journal of sound and vibration 257 (5), 869-886

[37] Rosi G., Madeo A., Guyader J.-L., 2013. Switch between fast and slow Biot compression waves induced by second gradient microstructur at material discontinuity surfaces in porous media. Int. J. Solids Struct., 50:10, 1721-1746

[38] Sanavia L., Schrefler B.A., Steinmann P., 2002. Geometrical and material non-linear analysis of fully and partially saturated porous media. in: Porous Media, pp. 341-381.

[39] G. Sciarra, F. dell'Isola, N. Ianiro and A. Madeo, 2008. A Variational Deduction of Second Gradient Poroelasticity I: General Theory. J. Mech. Materials Struct. 3 (3) 507-526.

[40] Scholtès, L., Hicher, P.Y., Nicot, F., Chareyre, B., Darve, F. 2009. On the capillary stress tensor in wet granular materials. Int. J. Numer. Anal. Meth. Geomech. 33 (10), 12891313.

[41] Scholtès, L., Chareyre, B., Nicot, F., Darve, F. 2009. Micromechanics of granular materials with capillary effects. Int J. Eng. Sc., 47:(11-12), 1460-1471.

[42] Yang Y., Misra A., 2012. Micromechanics based second gradient continuum theory for shear band modeling in cohesive granular materials following damage elasticity. International Journal of Solids and Structures, 49, $2500-2514$.

[43] Zubov L.M. , Eremeev V.A., 1996. Equations for a viscoelastic micropolar fluid. Physics-Doklady 41, 598-601 